\title{
Conditional Intensity and Gibbsianness of Determinantal Point Processes
}

\author{
Hans-Otto Georgii ${ }^{1}$ and Hyun Jae Yoo $^{2}$
}

Received January 26, 2004; accepted August 25, 2004

\begin{abstract}
The Papangelou intensities of determinantal (or fermion) point processes are investigated. These exhibit a monotonicity property expressing the repulsive nature of the interaction, and satisfy a bound implying stochastic domination by a Poisson point process. We also show that determinantal point processes satisfy the so-called condition $\left(\Sigma_{\lambda}\right)$, which is a general form of Gibbsianness. Under a continuity assumption, the Gibbsian conditional probabilities can be identified explicitly.
\end{abstract}

KEY WORDS: Determinantal point process; fermion point process; Gibbs point process; Papangelou intensity; stochastic domination; percolation.

\section{INTRODUCTION}

The aim of this paper is to investigate the dependence structure of determinantal (also called fermion) point processes, abbreviated DPP's. These are point processes on a locally compact metric space $E$ with a particular repulsive dependence structure induced by the fact that their correlation functions are given by suitable determinants. More explicitly, the correlation function $\rho(\alpha)$ at a finite configuration $\alpha$ is the determinant of the matrix obtained by evaluating a positive definite integral kernel at the points of $\alpha$ (see Section 2.1 for details).

Since their invention by Macchi, ${ }^{(13)}$ DPP's have attracted much interest from various viewpoints. Spohn ${ }^{(29,30)}$ investigated the dynamics of the so-called Dyson's model, a model of interacting Brownian particles on the

\footnotetext{
${ }^{1}$ Mathematisches Institut der Universität München, Theresienstraße 39, 80333 München, Germany; e-mail: georgii@lmu.de

${ }^{2}$ University College, Yonsei University, 134 Shinchon-dong, Seodaemoon-gu, Seoul 120-749, Korea; e-mail: yoohj@yonsei.ac.kr
} 
real line with pair force $1 / x$, or pair interaction $-\log x$. Its invariant measure is a typical DPP having the sine kernel as defining integral kernel. Rather recently, the theory of DPP's has been developed much further. Soshnikov ${ }^{(28)}$ established the full existence theorem for DPP's, discussed many examples occurring in various fields of physics and mathematics, and derived various further results for DPP's with translation-invariant kernels, including mixing properties and central limit theorems. Shirai and Takahashi ${ }^{(24)}$ also dealt with the existence theorem and extended the theory to some generalized DPP's including Boson processes. They also established some particular properties such as limit theorems, ergodic properties, and the Gibbs property of the corresponding discrete model. ${ }^{(25)}$ In a series of papers, Borodin and Olshanski studied the DPP's appearing in the representation of the infinite symmetric group (see ref. 3 and the references therein). Lyons and Steif ${ }^{(11,12)}$ investigated the ergodic and stochastic domination properties of DPP's on discrete lattices. The Glauber dynamics leaving some DPP's invariant was studied by Shirai and the second author. ${ }^{(26)}$

In this paper we ask for the dependence properties of DPP's. Our leading questions are the following:

- What can be said about the repulsive nature of the interaction?

- When are determinantal point processes Gibbsian?

Unfortunately, to answer these questions we need to exclude the interesting case when 1 belongs to the spectrum of the underlying integral operator, and in general we can only establish a weak form of Gibbsianness. To prove full Gibbsianness we need to impose a natural though restrictive continuity assumption. A key quantity we consider is the conditional intensity in the sense of Papangelou, which is a function $c(x, \xi)$ of points $x \in E$ and configurations $\xi$. Its intuitive meaning is that, for a suitable reference measure $\lambda$ on $E, c(x, \xi) \lambda(d x)$ is the conditional probability of having a particle in $d x$ when the configuration $\xi$ is given. We will show that, locally on relatively compact regions, Papangelou intensities always exist, are given by ratios of determinants, and are decreasing functions of $\xi$ (Theorem 3.1). This monotonicity is a natural expression of the repulsiveness of DPP's. In particular, it implies negative correlations of local vacuum events stating that some local regions contain no particles (Corollary 3.3). We also show that the local Papangelou intensities are bounded from above. This implies stochastic domination of DPP's by Poisson point processes and, for $E=\mathbb{R}^{d}$, the absence of percolation in the associated Boolean model when the underlying integral kernel is small enough (Corollaries 3.4 and 3.5). 
Next, we will show that the Papangelou intensities of DPP's exist not only locally but also globally (Theorem 3.6). This means that all DPP's are Gibbsian in a general sense, in that one can write down natural versions of their conditional distributions in local regions, when the configuration outside of this region is fixed, and these conditional distributions are absolutely continuous with respect to the Poisson point process. It is a more difficult question to decide whether the associated conditional Hamiltonians can be expressed in terms of the underlying integral kernel in the natural way one expects. Here we only have a partial result, stating that this holds, whenever these natural versions are continuous almost everywhere (Theorem 3.7). The last condition holds in particular, when $E=\mathbb{R}^{d}$ and the associated interaction kernel has finite range and is continuous and small enough (Proposition 3.9).

In Section 2 we set up the stage. That is, in Section 2.1 we recall the definition of DPP's together with some basic facts, while Section 2.2 contains a discussion of the Papangelou intensity and its significance. Our results are stated in Section 3. The proofs follow in Section 4. In Appendix A we collect some useful facts and discuss in particular the question of how to make a proper choice of integral kernels.

\section{PRELIMINARIES}

\subsection{Determinantal Point Processes}

In this section, we describe the general setting and recall the definition of DPP's. For a more complete account of DPP's we refer to the survey ${ }^{(28)}$ and the articles. ${ }^{(11,12,24,25)}$ Let $E$ be any locally compact metric space serving as the state space of the points, $\mathcal{B}$ the Borel $\sigma$-algebra on $E$, and $\mathcal{B}_{0}$ the system of all relatively compact Borel sets in $E$. Also, let $\lambda$ be a diffuse, locally finite reference measure on $(E, \mathcal{B})$. The standard case is when $E=\mathbb{R}^{d}$ and $\lambda$ is Lebesgue measure.

Let $\mathcal{X}$ be the space of all locally finite subsets (configurations) in $E$, i.e.,

$$
\mathcal{X}:=\left\{\xi \subset E:|\xi \cap \Lambda|<\infty \text { for all } \Lambda \in \mathcal{B}_{0}\right\}
$$

where $|A|$ denotes the cardinality of a set $A$. Later on, we will exploit the fact that $\mathcal{X}$ is partially ordered by inclusion. Given any $\Lambda \subset E$, we write $\mathcal{X}_{\Lambda}:=\{\xi \in \mathcal{X}: \xi \subset \Lambda\}$ for the set of all configurations in $\Lambda$, and $r_{\Lambda}: \xi \rightarrow \xi_{\Lambda}:=\xi \cap \Lambda$ for the corresponding projection from $\mathcal{X}$ onto $\mathcal{X}_{\Lambda}$. Also let $N_{\Lambda}: \xi \rightarrow|\xi \cap \Lambda|$ be the associated counting variable on $\mathcal{X}$, and $\mathcal{F}_{\Lambda}$ be the smallest $\sigma$-algebra on $\mathcal{X}$ such that $N_{\Delta}$ is measurable for all relatively 
compact Borel sets $\Delta \subset \Lambda$. We write $\mathcal{F}$ for $\mathcal{F}_{E}$. Each configuration $\xi \in \mathcal{X}$ can be identified with the integer-valued Radon measure $\sum_{x \in \xi} \delta_{x}$ on the Borel $\sigma$-algebra on $E$. The vague topology for the latter then induces a topology on $\mathcal{X}$ turning $\mathcal{X}$ into a Polish space. It is well known that $\mathcal{F}$ is the associated Borel $\sigma$-algebra. ${ }^{(4,23)}$ A point process is a probability measure $\mu$ on $(\mathcal{X}, \mathcal{F})$. We write $\mu_{\Lambda}:=\mu \circ r_{\Lambda}^{-1}$ for its marginal on $\mathcal{X}_{\Lambda}$.

Next let $\mathcal{X}_{0}=\{\alpha \in \mathcal{X}:|\alpha|<\infty\}$ be the set of all finite configurations in $E . \mathcal{X}_{0}$ is equipped with the trace $\sigma$-algebra $\mathcal{F}_{0}=\mathcal{F} \mid \mathcal{X}_{0}$ and the $\lambda$-sample measure $L$ defined by the identity

$$
\int_{\mathcal{X}_{0}} f(\alpha) L(d \alpha)=\sum_{m=0}^{\infty} \frac{1}{m !} \int_{E^{m}} f\left(\left\{x_{1}, \ldots, x_{m}\right\}\right) \lambda\left(d x_{1}\right) \cdots \lambda\left(d x_{m}\right)
$$

for any measurable function $f: \mathcal{X}_{0} \rightarrow \mathbb{R}_{+}$. For any $\Lambda \subset E$ we let $L_{\Lambda}(d \alpha)=$ $1_{\{\alpha \subset \Lambda\}} L(d \alpha)$ be the restriction of $L$ to $\mathcal{X}_{\Lambda}$. Here we use the notation $1_{A}$ for the indicator function of a set $A$.

Recall that a point process $\mu$ is said to have the correlation function $\rho: \mathcal{X}_{0} \rightarrow \mathbb{R}_{+}$if $\rho$ is measurable and satisfies

$$
\int_{\mathcal{X}} \sum_{\alpha \in \mathcal{X}_{0}: \alpha \subset \xi} u(\alpha) \mu(d \xi)=\int_{\mathcal{X}_{0}} u(\alpha) \rho(\alpha) L(d \alpha)
$$

for any measurable $u: \mathcal{X}_{0} \rightarrow \mathbb{R}_{+}$. (For a general account of the interrelationship $\mu \leftrightarrow \rho$ between point processes and correlation functions we refer to the recent paper. ${ }^{(9)}$ ) The Poisson point process $\pi^{z}$ with (locally integrable) intensity function $z: E \rightarrow[0, \infty[$ is the unique point process having correlation function $\rho^{z}(\alpha)=\prod_{x \in \alpha} z(x)$. Equivalently, $\pi^{z}$ is the unique point process such that, for each $\Lambda \in \mathcal{B}_{0}$, the projection $\pi_{\Lambda}^{z}$ has the RadonNikodym density $\xi \rightarrow e^{-\int_{\Lambda} z d \lambda} \prod_{x \in \xi} z(x)$ relative to $L_{\Lambda}$. The characteristic feature of the determinantal point processes to be considered here is that their correlation function is given by suitable determinants. Given a function $K: E \times E \rightarrow \mathbb{C}$, we write

$$
K(\alpha, \alpha)=(K(x, y))_{x, y \in \alpha}
$$

for the matrix obtained by evaluating $K$ at a finite configuration $\alpha \in \mathcal{X}_{0}$.

Definition 2.1. Let $K(x, y), x, y \in E$, be the integral kernel of a positive $^{3}$ Hermitian integral operator $K$ on $L^{2}(E, \lambda)$. A point process $\mu$ with

\footnotetext{
${ }^{3}$ We use the terms "positive operator" and "positive definite matrix" always in the weak sense, in that 0 may belong to the spectrum.
} 
correlation function

$$
\rho(\alpha)=\operatorname{det} K(\alpha, \alpha)
$$

is called a determinantal point process (abbreviated DPP) with correlation kernel $K$.

The DPP's defined above are also known as fermion point processes; see ref. 24 for the boson case, where the determinant is replaced by the permanent, and more general determinantal processes. In fact, there exist interesting examples of DPP's for non-Hermitian kernels such as the discrete Bessel kernel (see ref. 2, for example). In this paper, however, we confine ourselves to the Hermitian case. On the technical side, we note that the matrices (2.3) involve the values of the integral kernel $K(x, y)$ not only for $\lambda^{\otimes 2}$-almost all $(x, y) \in E^{2}$ but also on the diagonal of $E^{2}$, which is a $\lambda^{\otimes 2}$-nullset because $\lambda$ is diffuse. So one needs to make a proper choice of $K(x, y)$, which is positive definite a.e., in that $K(\alpha, \alpha)$ is positive definite for $L$-almost all $\alpha \in \mathcal{X}_{0}$ (implying that the correlation function $\rho$ is indeed non-negative $L$-a.e.). In Lemma A.4 it will be explained how this can be done.

Concerning the existence of DPP's, we state the following theorem from ref. 28, (see also refs. 4, 13 and 24). I stands for the identity operator, and the ordering $S \leqslant T$ between operators means that $T-S$ is a positive operator.

Theorem 2.2 (Macchi, Soshnikov). A Hermitian locally trace class operator $K$ on $L^{2}(E, \lambda)$ defines a DPP $\mu$ if and only if $0 \leqslant K \leqslant I$, and in this case $\mu$ is unique.

Any DPP $\mu$ is locally absolutely continuous with respect to the $\lambda$-sample measure $L$ and admits explicit expressions for the local densities. To be specific, for each $\Lambda \in \mathcal{B}_{0}$ let $P_{\Lambda}: L^{2}(E, \lambda) \rightarrow L^{2}\left(\Lambda, \lambda_{\Lambda}\right)$ be the projection operator and $K_{\Lambda}:=P_{\Lambda} K P_{\Lambda}$ the restriction of $K$ onto $L^{2}\left(\Lambda, \lambda_{\Lambda}\right)$. That is, $K_{\Lambda}$ admits the kernel $K_{\Lambda}(x, y)=1_{\Lambda}(x) K(x, y) 1_{\Lambda}(y)$. Suppose that $\mu$ is the unique DPP for an operator $K$ as in Theorem 2.2. Then the density function of $\mu_{\Lambda}$ with respect to $L_{\Lambda}$, the so-called Janossy density ${ }^{(4)}$ of $\mu$, is given by $(24,28)$

$$
\sigma_{\Lambda}(\xi)=\operatorname{det}\left(I-K_{\Lambda}\right) \operatorname{det} J_{[\Lambda]}(\xi, \xi), \quad \xi \in \mathcal{X}_{\Lambda}
$$

here $J_{[\Lambda]}:=K_{\Lambda}\left(I-K_{\Lambda}\right)^{-1}$, and the normalization constant $\operatorname{det}\left(I-K_{\Lambda}\right)$ is to be understood as a Fredholm determinant. ${ }^{(27)}$ A priori, this formula requires that $0 \leqslant K_{\Lambda}<I$. However, one can show that (2.4) still makes 
sense when $\left\|K_{\Lambda}\right\|=1$ (see ref. 28, p. 935). In view of (2.4), we call $J_{[\Lambda]}$ the local interaction operator and its kernel the local interaction kernel for $\Lambda$. Again, Lemma A.3 allows to choose the kernel $J_{[\Lambda]}$ in such a way that all matrices $J_{[\Lambda]}(\xi, \xi)$ are positive definite. Finally, we note that the correlation function $\rho$ can be recovered from the local densities $\left(\sigma_{\Lambda}\right)$ by

$$
\rho(\alpha)=\int_{\mathcal{X}_{\Lambda}} \sigma_{\Lambda}(\alpha \xi) L_{\Lambda}(d \xi)
$$

for $\alpha \in \mathcal{X}_{\Lambda}$, where $\alpha \xi$ is shorthand for $\alpha \cup \xi$.

\subsection{Papangelou Intensities}

Here we summarize some facts concerning the reduced (compound) Campbell measure of a point process $\mu$ as well as its Papangelou intensity, which describes the local dependence of particles.

Definition 2.3. (a) The reduced (or modified) Campbell measure of a point process $\mu$ is the measure $C_{\mu}$ on the product space $(E \times \mathcal{X}, \mathcal{B} \otimes \mathcal{F})$ defined by

$$
C_{\mu}(A)=\int \mu(d \xi) \sum_{x \in \xi} 1_{A}(x, \xi \backslash x), \quad A \in \mathcal{B} \otimes \mathcal{F}
$$

where $\xi \backslash x:=\xi \backslash\{x\}$.

(b) The reduced compound Campbell measure of $\mu$ is the measure $\widehat{C}_{\mu}$ on the product space $\left(\mathcal{X}_{0} \times \mathcal{X}, \mathcal{F}_{0} \otimes \mathcal{F}\right)$ satisfying

$$
\widehat{C}_{\mu}(B)=\int \mu(d \xi) \sum_{\alpha \in \mathcal{X}_{0}: \alpha \subset \xi} 1_{B}(\alpha, \xi \backslash \alpha), \quad B \in \mathcal{F}_{0} \otimes \mathcal{F}
$$

It is well known and easy to check that the reduced Campbell measure of the Poisson point process $\pi^{z}$ is given by $C_{\pi^{z}}(d x, d \xi)=z(x) \lambda(d x) \pi^{z}(d \xi)$, and a classical result of Mecke ${ }^{(15)}$ states that $\pi^{z}$ is the only point process with this property. This fact suggests the following concept.

Definition 2.4. A point process $\mu$ is said to satisfy condition $\left(\Sigma_{\lambda}\right)$ if $C_{\mu} \ll \lambda \otimes \mu$. Any Radon-Nikodym density $c$ of $C_{\mu}$ relative to $\lambda \otimes \mu$ is then called (a version of) the Papangelou (conditional) intensity (abbreviated PI) of $\mu$. 
More explicitly, $c$ is a PI of $\mu$ if

$$
\int \mu(d \xi) \sum_{x \in \xi} f(x, \xi \backslash x)=\int \lambda(d x) \int \mu(d \xi) c(x, \xi) f(x, \xi)
$$

for all measurable functions $f: E \times \mathcal{X} \rightarrow \mathbb{R}_{+}$. Another way of stating this is that the intensity measure of $\mu$ has a density $\rho_{1}$ relative to $\lambda$, and the reduced Palm distribution $\mu^{x}$ of $\mu$ at $x$ (which is defined by the disintegration formula $\left.C_{\mu}(d x, d \xi)=\lambda(d x) \rho_{1}(x) \mu^{x}(d \xi)\right)$ is absolutely continuous with respect to $\mu$ with density $c(x, \cdot) / \rho_{1}(x)$ for $\lambda$-a.a. $x \in\left\{\rho_{1}>0\right\}$. Intuitively, $c(x, \xi) \lambda(d x)$ is the conditional probability for a particle in the differential region $d x$ given the configuration $\xi$. Also, if

$$
G h(\xi)=\int \lambda(d x) c(x, \xi)[h(\xi \cup x)-h(\xi)]+\sum_{x \in \xi}[h(\xi \backslash x)-h(\xi)]
$$

is the formal generator of a birth-and-death process with birth rate $c(x, \xi) \lambda(d x)$ for a particle in $d x$ and death rate 1 for each particle, (2.5) is equivalent to the reversibility equation $\int g G h d \mu=\int h G g d \mu$; to see this it is sufficient to set $f(x, \xi)=g(\xi) h(\xi \cup x)$.

The following remark lists some further consequences of condition $\left(\Sigma_{\lambda}\right)$. In particular, it shows that $\left(\Sigma_{\lambda}\right)$ processes are Gibbsian in a general sense.

Remark 2.5. For any $\mu$ satisfying condition $\left(\Sigma_{\lambda}\right)$ the following conclusions hold:

(a) The reduced compound Campbell measure $\widehat{C}_{\mu}$ is absolutely continuous with respect to $L \otimes \mu$ with a Radon-Nikodym density $\widehat{c}$ satisfying $\widehat{c}(\emptyset, \xi)=1$ and

$$
\widehat{c}(\alpha, \xi)=c\left(x_{1}, \xi\right) \prod_{i=2}^{n} c\left(x_{i}, x_{1} \ldots x_{i-1} \xi\right), \quad \text { when } \alpha=\left\{x_{1}, \ldots, x_{n}\right\}
$$

here $x_{1} \ldots x_{i-1} \xi=\left\{x_{1}, \ldots, x_{i-1}\right\} \cup \xi$. In particular, the right-hand side of (2.6) is almost surely symmetric in $x_{1}, \ldots, x_{n} . \widehat{c}$ is called the compound Papangelou intensity (CPI). Explicitly, the relation $\widehat{c}=d \widehat{C}_{\mu} / d(L \otimes \mu)$ means that

$$
\int \mu(d \xi) \sum_{\alpha \in \mathcal{X}_{0}: \alpha \subset \xi} f(\alpha, \xi \backslash \alpha)=\int L(d \alpha) \int \mu(d \xi) \widehat{c}(\alpha, \xi) f(\alpha, \xi)
$$


for any measurable $f: \mathcal{X}_{0} \times \mathcal{X} \rightarrow \mathbb{R}_{+}$, and follows easily from (2.5) by induction on $|\alpha|$.

(b) For an $f$ depending only on $\alpha$, a comparison of (2.7) and (2.2) shows that the correlation function and the CPI of $\mu$ are related to each other by

$$
\rho(\alpha)=\int \mu(d \xi) \widehat{c}(\alpha, \xi) \text { for } L \text {-almost all } \alpha
$$

(c) Let $\Lambda \in \mathcal{B}_{0}$. Applying (2.7) to $f(\alpha, \xi)=g(\alpha) h(\xi) 1_{\left\{\alpha \subset \Lambda, N_{\Lambda}(\xi)=0\right\}}$ for any $\mathcal{F}_{\Lambda}$-measurable $g: \mathcal{X} \rightarrow \mathbb{R}_{+}$and $\mathcal{F}_{\Lambda^{c}}$-measurable $h: \mathcal{X} \rightarrow \mathbb{R}_{+}$and taking conditional expectations we find

$$
\begin{aligned}
& \int \mathbb{E}_{\mu}\left(g \mid \mathcal{F}_{\Lambda^{c}}\right) h d \mu=\int g h d \mu=\int f d \widehat{C}_{\mu} \\
& =\int \mu(d \xi) h(\xi) \mu\left(N_{\Lambda}=0 \mid \mathcal{F}_{\Lambda^{c}}\right)(\xi) \int L_{\Lambda}(d \alpha) g(\alpha) \widehat{c}\left(\alpha, \xi_{\Lambda^{c}}\right) .
\end{aligned}
$$

Hence

$$
\mathbb{E}_{\mu}\left(g \mid \mathcal{F}_{\Lambda^{c}}\right)(\xi)=\mu\left(N_{\Lambda}=0 \mid \mathcal{F}_{\Lambda^{c}}\right)(\xi) \int g \widehat{c}\left(\cdot, \xi_{\Lambda^{c}}\right) d L_{\Lambda}
$$

for $\mu$-almost all $\xi$ and any $\mathcal{F}_{\Lambda}$-measurable $g$. In particular, for $g \equiv 1$, we find that $\mu\left(N_{\Lambda}=0 \mid \mathcal{F}_{\Lambda^{c}}\right)>0$ almost surely for each $\Lambda \in \mathcal{B}_{0}$, a property introduced by Papangelou ${ }^{(19)}$ as condition $(\Sigma)$, and by $\operatorname{Kozlov}^{(10)}$ as the condition of non-degenerate vacuum.

The observations in the preceding remark are due to Matthes, Warmuth and Mecke ref. (14, Section 3) and give one part of their theorem below (cf. also ref. 17, Theorem 2').

Theorem 2.6 (Matthes, Warmuth and Mecke). A point process $\mu$ satisfies condition $\left(\Sigma_{\lambda}\right)$ with PI $c$ if and only if, for each $\Lambda \in \mathcal{B}_{0}, \mu$ is absolutely continuous relative to $L_{\Lambda} \otimes \mu_{\Lambda^{c}}$ with a density $\gamma_{\Lambda}$ satisfying $\gamma_{\Lambda}(x \xi)=0$ whenever $\gamma_{\Lambda}(\xi)=0$ and $x \in \Lambda \backslash \xi$. In this case, for $L_{\Lambda} \otimes \mu_{\Lambda^{c-}}$ almost all $\xi \in \mathcal{X}$ we have

$$
\gamma_{\Lambda}(\xi)=Z_{\Lambda}\left(\xi_{\Lambda^{c}}\right)^{-1} \widehat{c}\left(\xi_{\Lambda}, \xi_{\Lambda^{c}}\right)
$$

with $0<Z_{\Lambda}\left(\xi_{\Lambda^{c}}\right)=\int \widehat{c}\left(\cdot, \xi_{\Lambda^{c}}\right) d L_{\Lambda}<\infty$, and $c(x, \xi)=\gamma_{\Lambda}(x \xi) / \gamma_{\Lambda}(\xi)$ for $\lambda \otimes \mu$-almost all $(x, \xi) \in \Lambda \times \mathcal{X}$. 
More explicitly, Eq. (2.9) means that for each bounded measurable function $f: \mathcal{X} \rightarrow \mathbb{R}$, the conditional expectation $\mathbb{E}_{\mu}\left(f \mid \mathcal{F}_{\Lambda^{c}}\right)(\xi)$ has the version

$$
G_{\Lambda}(f \mid \xi):=Z_{\Lambda}\left(\xi_{\Lambda^{c}}\right)^{-1} \int f\left(\alpha \xi_{\Lambda^{c}}\right) \widehat{c}\left(\alpha, \xi_{\Lambda^{c}}\right) L_{\Lambda}(d \alpha)
$$

Remark 2.7. Theorem 2.6 has a counterpart for the restriction of a point process $\mu$ to a local region $\Lambda \in \mathcal{B}_{0}$. Let $\lambda_{\Lambda}$ be the restriction of $\lambda$ to the $\sigma$-algebra $\mathcal{B}_{\Lambda}$ of Borel subsets of $\Lambda$. Then $\mu_{\Lambda}$ satisfies condition $\left(\Sigma_{\lambda_{\Lambda}}\right)$ with a PI $c_{\Lambda}$ if and only if $\mu_{\Lambda}$ is absolutely continuous with respect to $L_{\Lambda}$ with a density $\sigma_{\Lambda}$ having an increasing zero-set $\left\{\sigma_{\Lambda}=0\right\}$. In this case, $\sigma_{\Lambda}=\sigma_{\Lambda}(\emptyset) \widehat{c}_{\Lambda}(\cdot, \emptyset) L_{\Lambda}$-almost everywhere, and $c_{\Lambda}(x, \xi)=\sigma_{\Lambda}(x \xi) / \sigma_{\Lambda}(\xi)$ for $\lambda_{\Lambda} \otimes \mu_{\Lambda}$ almost all $(x, \xi) \in \Lambda \times \mathcal{X}_{\Lambda}$. This follows from the above by noting that $\mu_{\Lambda}$, regarded as a measure on $(\mathcal{X}, \mathcal{F})$ supported on $\left\{N_{\Lambda^{c}}=0\right\}$, is trivial on $\mathcal{F}_{\Lambda^{c}}$ (cf. also ref. 5, Proposition 3.1).

\section{RESULTS}

Our results on DPP's are based on the following standing assumption on the underlying correlation operator $K$.

Hypothesis $\mathbf{( H )} . \quad K$ is a Hermitian integral operator on $L^{2}(E, \lambda)$ such that

(a) $K$ is of local trace class, and

(b) $\operatorname{spec} K \subset[0,1[$; i.e., $0 \leqslant K \leqslant I$ in the operator ordering, and $\|K\|<1$.

We write $\mu$ for the unique DPP with correlation kernel $K$.

Let us comment on these assumptions. First, $K$ is necessarily a Carleman operator, ${ }^{(8)}$ in that its integral kernel satisfies $K(x, \cdot) \in L^{2}(E, \lambda)$ for $\lambda$-almost all $x \in E$; this will be shown in Lemma A.4(b). Second, it follows from (b) that $J:=K(I-K)^{-1}$ exists as a bounded operator; we call $J$ the (global) interaction operator. Since the Carleman operators form a right ideal in the space of bounded operators on $L^{2}(E, \lambda)$ (ref. 8, Theorem 11.6), hypothesis $(\mathrm{H})$ ensures that $J$ is also a Carleman operator. Moreover, since $K$ is supposed to be of local trace class, so is $J$; for, if $\Lambda \in \mathcal{B}_{0}$ then $J_{\Lambda}:=P_{\Lambda} J P_{\Lambda} \leqslant(1-\|K\|)^{-1} K_{\Lambda}$, hence $\operatorname{Tr} J_{\Lambda} \leqslant(1-\|K\|)^{-1} \operatorname{Tr} K_{\Lambda}<$ $\infty$. Assumption (a) implies further that, for each $\Lambda \in \mathcal{B}_{0}$, the local operators $K_{\Lambda}$ and $J_{\Lambda}$ as well as the local interaction operator $J_{[\Lambda]}:=K_{\Lambda}(I-$ $\left.K_{\Lambda}\right)^{-1}$ satisfy the conditions of Lemma A.3. So, their kernel functions can 
and will be chosen in such a way that all associated matrices $K_{\Lambda}(\alpha, \alpha)$, $J_{\Lambda}(\alpha, \alpha)$ and $J_{[\Lambda]}(\alpha, \alpha)$ defined in (2.3) are positive definite. Finally, we note that necessarily $0 \in \operatorname{spec} K$ because $K_{\Lambda}$, being trace class, is compact and thus has eigenvalues tending to zero.

Our first result describes the local behavior of DPP's under $(\mathrm{H})$.

Theorem 3.1. For each $\Lambda \in \mathcal{B}_{0}, \mu_{\Lambda}$ satisfies condition $\left(\Sigma_{\lambda_{\Lambda}}\right)$, and a version of its CPI $\widehat{c}_{\Lambda}$ is given by

$$
\widehat{c}_{\Lambda}(\alpha, \xi)=\operatorname{det} J_{[\Lambda]}(\alpha \xi, \alpha \xi) / \operatorname{det} J_{[\Lambda]}(\xi, \xi) ;
$$

the ratio is defined to be zero whenever the denominator vanishes. This version also satisfies the inequalities

$$
\widehat{c}_{\Lambda}(\alpha, \xi) \geqslant \widehat{c}_{\Lambda}(\alpha, \eta) \text { and } 0 \leqslant \widehat{c}_{\Lambda}(\alpha, \xi) \leqslant \operatorname{det} J_{[\Lambda]}(\alpha, \alpha) \leqslant \prod_{x \in \alpha} J_{[\Lambda]}(x, x),
$$

whenever $\xi \subset \eta$ and $\alpha \subset \Lambda \backslash \eta$.

Here are some consequences of the theorem. Let us look first at the local CPI $\widehat{c}_{\Lambda}$ in (3.1). Let $\Lambda, \Delta \in \mathcal{B}_{0}$ with $\Lambda \subset \Delta$ be given, and $f: \mathcal{X}_{\Lambda} \rightarrow \mathbb{R}_{+}$ an arbitrary measurable function. Then, combining (3.1) with Remark 2.7 and (2.10) we find that

$$
\begin{aligned}
\mathbb{E}_{\mu}\left(f \mid \mathcal{F}_{\Delta \backslash \Lambda}\right)(\xi) & =G_{\Lambda, \Delta}(f \mid \xi) \\
& :=Z_{\Lambda, \Delta}\left(\xi_{\Delta \backslash \Lambda}\right)^{-1} \int L_{\Lambda}(d \alpha) f(\alpha) \widehat{c}_{\Delta}\left(\alpha, \xi_{\Delta \backslash \Lambda}\right)
\end{aligned}
$$

for $\mu$-almost all $\xi \in \mathcal{X}$. Let us comment on these conditional probabilities.

Remark 3.2. (a) For any $\xi \in \mathcal{X}_{\Delta \backslash \Lambda}$, the normalization constant

$$
Z_{\Lambda, \Delta}(\xi)=\int L_{\Lambda}(d \alpha) \widehat{c}_{\Delta}(\alpha, \xi)
$$

is finite. In fact, $Z_{\Lambda, \Delta}(\xi) \leqslant \operatorname{det}\left(I+J_{\Lambda}\right)$. Moreover, $Z_{\Lambda, \Delta}(\xi)$ is nonzero, whenever $\operatorname{det} J_{[\Delta]}(\xi, \xi)>0$ because $L_{\Lambda}(\{\emptyset\})=1$. This means that $G_{\Lambda, \Delta}(\cdot \mid \xi)$ is a well-defined probability measure on $\mathcal{X}_{\Lambda}$ for all such $\xi$.

(b) For all $\xi$ with $D:=\operatorname{det} J_{[\Delta]}(\xi, \xi)>0, G_{\Lambda, \Delta}(\cdot \mid \xi)$ is a DPP with interaction kernel $J_{\Lambda, \Delta}^{\xi}(x, y):=D^{-1} \operatorname{det} J_{[\Delta]}(x \xi, y \xi), x, y \in \Lambda$. (The last matrix is defined in obvious analogy to (2.3).) This observation is analogous to the fact observed in ref. 24 that the reduced Palm measures $\mu^{x}$ of $\mu$ are again determinantal with correlation kernel $K^{x}\left(y, y^{\prime}\right)=$ $K(x, x)^{-1} \operatorname{det} K\left(y x, y^{\prime} x\right)$. 
Turning to the inequalities (3.2), we emphasize that the monotonicity of the functions $\widehat{c}_{\Lambda}(\alpha, \cdot)$ expresses the repulsive nature of the particle interaction in an infinitesimal way. As a matter of fact, this monotonicity is analogous to the inequality

$$
\rho\left(\alpha_{1}\right) \rho\left(\alpha_{2}\right) \geqslant \rho\left(\alpha_{1} \cup \alpha_{2}\right) \rho\left(\alpha_{1} \cap \alpha_{2}\right), \quad \alpha_{1}, \alpha_{2} \in \mathcal{X}_{0},
$$

derived in ref. 24 for the correlation function $\rho$ of $\mu$, and follows in the same way from an inequality for determinants of Hermitian matrices (see (A.2)). The following corollary provides an integral version of this repulsiveness.

Corollary 3.3. For any $\Lambda \subset \Delta \in \mathcal{B}_{0}$ and any measurable function $f: \mathcal{X}_{\Lambda} \rightarrow \mathbb{R}_{+}$, the ratio $G_{\Lambda, \Delta}(f \mid \xi) / G_{\Lambda, \Delta}\left(N_{\Lambda}=0 \mid \xi\right)$ is decreasing in $\xi \in$ $\mathcal{X}_{\Delta \backslash \Lambda}$. In particular, $G_{\Lambda, \Delta}\left(N_{\Lambda}=0 \mid \cdot\right)$ is increasing, and for $\Lambda \subset \Delta_{1} \subset \Delta_{2} \in \mathcal{B}_{0}$ we have

$$
\mu\left(N_{\Lambda}=0 \mid N_{\Delta_{2} \backslash \Lambda}=0\right) \leqslant \mu\left(N_{\Lambda}=0 \mid N_{\Delta_{1} \backslash \Lambda}=0\right) \leqslant \mu\left(N_{\Lambda}=0\right) .
$$

The statement of the corollary is weaker than one may hope. In fact, one might guess that $G_{\Lambda, \Delta}(f \mid \xi)$ is decreasing in $\xi$ for any increasing $\mathcal{F}_{\Lambda}$-measurable function $f$. (The corollary implies this assertion only for $f=1_{\left\{N_{\Lambda} \geqslant 1\right\}}$.) However, we have some doubts, whether this can be expected to hold in general. For, if $f$ depends only on some part $\Lambda_{0}$ of $\Lambda$, then an increase of $\xi$ may repel some particles from $\Lambda \backslash \Lambda_{0}$, giving a chance to some additional particles in $\Lambda_{0}$, so that $G_{\Lambda, \Delta}(f \mid \xi)$ will increase. So, the situation is less satisfactory for point processes than in the discrete case (cf. Theorem 6.5 of ref. 11). Nevertheless, (3.4) implies that, for disjoint $\Lambda, \Delta \in \mathcal{B}_{0}$, the events $\left\{N_{\Lambda}=0\right\}$ and $\left\{N_{\Delta}=0\right\}$ are negatively correlated (see Proposition 2.7 of ref. 25 for the corresponding result in the discrete case).

Next we exploit the domination bound $c_{\Lambda}(x, \xi) \leqslant J_{[\Lambda]}(x, x)$, which will imply that $\mu$ is stochastically dominated by a Poisson process. Recall that a point process $v$ is stochastically dominated by a point process $v^{\prime}$, written $v \preceq v^{\prime}$, if

$$
\int f d v \leqslant \int f d v^{\prime}
$$

for every increasing measurable function $f: \mathcal{X} \rightarrow \mathbb{R}$. Here, $f$ is said to be increasing if $f(\xi) \leqslant f(\eta)$, whenever $\xi \subset \eta$. The intensity function of the dominating Poisson process shall be given by $z(x)=J(x, x)$, where $J(x, y)$ is a properly chosen integral kernel of $J$. To understand the necessity of a 
proper choice one should note again that the diagonal in $E^{2}$ is a $\lambda^{\otimes 2}$-nullset because $\lambda$ is diffuse; the behavior of the integral kernel on the diagonal that enters in the definition of $z(x)$ is therefore a priori unrelated to the operator $J$ (except when the kernel is continuous, which we do not require here). However, a natural way of determining the kernel function on the diagonal is in terms of the local trace formula

$$
\operatorname{Tr}\left(J_{\Lambda}\right)=\int_{\Lambda} J(x, x) \lambda(d x) \text { for all } \Lambda \in \mathcal{B}_{0}
$$

and such a choice is possible according to Lemma A.4. The function $z(x):=J(x, x)$ is then locally $\lambda$-integrable, and the Poisson point process $\pi^{J}$ with intensity function $z(x)$ is well defined.

Corollary 3.4. $\mu \preceq \pi^{J}$.

In the case when $E=\mathbb{R}^{d}$ and $\lambda$ is Lebesgue measure, the last result implies in particular that there is no percolation in the Boolean model associated to $\mu$, when $J(x, x)$ is small enough. Let $b_{R}(x)$ denote the closed ball of radius $R<\infty$ in $\mathbb{R}^{d}$ centered at $x$, and for $\xi \in \mathcal{X}$ let

$$
B_{R}(\xi)=\bigcup_{x \in \xi} b_{R}(x)
$$

the associated Boolean set. $B_{R}(\xi)$ splits into connected components called clusters. A cluster is called infinite, if it consists of infinitely many points of $\xi$, or equivalently, if its diameter is infinite. It is well known ${ }^{(7,16)}$ that, for $d \geqslant 2$, there exists a critical threshold $0<z_{c} \equiv z_{c}(d, R)<\infty$ for Poisson percolation: for the Poisson point process $\pi^{z}$ with constant intensity function $z>0$, one has

$$
\pi^{z}\left(\exists \text { infinite cluster of } B_{R}(\cdot)\right)= \begin{cases}0 & \text { if } z<z_{c} \\ 1 & \text { if } z>z_{c}\end{cases}
$$

For $d=1$ one has $z_{c}=+\infty$ (ref. 16, Theorem 3.1).

Corollary 3.5. Let $E=\mathbb{R}^{d}$ and $\lambda$ be Lebesgue measure. If

$$
z(J):=\limsup _{|x| \rightarrow \infty} J(x, x)<z_{c}
$$

then $\mu\left(\exists\right.$ infinite cluster of $\left.B_{R}(\cdot)\right)=0$. 
We now address the question of whether DPP's are Gibbsian. In view of Remark 2.5 and Theorem 2.6, the following result implies that $\mu$ is Gibbsian at least in a general sense.

Theorem 3.6. $\mu$ satisfies condition $\left(\Sigma_{\lambda}\right)$. Its CPI is given by

$$
\widehat{c}(\alpha, \xi)=\lim _{n \rightarrow \infty} \widehat{c}_{\Delta_{n}}\left(\alpha, \xi_{\Delta_{n}}\right) \text { for } L \otimes \mu \text {-almost all }(\alpha, \xi)
$$

where $\widehat{c}_{\Delta_{n}}$ is given by (3.1) and $\left(\Delta_{n}\right)$ is any sequence in $\mathcal{B}_{0}$ that increases to $E$. In particular, $\mu\left(N_{\Lambda}=0 \mid \mathcal{F}_{\Lambda^{c}}\right)>0 \mu$-almost surely for each $\Lambda \in \mathcal{B}_{0}$ and, for any $\mathcal{F}_{\Lambda}$-measurable $f: \mathcal{X} \rightarrow \mathbb{R}_{+}$, the ratio

$$
\mathbb{E}_{\mu}\left(f \mid \mathcal{F}_{\Lambda^{c}}\right) / \mu\left(N_{\Lambda}=0 \mid \mathcal{F}_{\Lambda^{c}}\right)
$$

of conditional expectations has a decreasing version.

Two remarks are in order. First, in view of the comments in Remark 3.2(b) and (2.5), the first assertion can be restated as an absolute continuity between DPP's: the DPP $\mu^{x}$ with correlation kernel $K^{x}$ is absolutely continuous with respect to the DPP $\mu$ for $K$ with density $c(x, \cdot) / K(x, x)$. Next, and more importantly, combining (3.6) with (3.1) and (2.10) we obtain at least an implicit formula for the conditional probabilities of $\mu$ given the events outside of a local region. But the question remains of whether the CPI, and thereby the conditional probabilities of $\mu$ can be identified in a more specific way. In fact, if we make a proper choice of the integral kernel $J(x, y)$ as in Lemma A.4, a natural candidate for $\widehat{c}$ is

$$
\widehat{c}_{*}(\alpha, \xi)=\lim _{\Delta \uparrow E} \operatorname{det} J\left(\alpha \xi_{\Delta}, \alpha \xi_{\Delta}\right) / \operatorname{det} J\left(\xi_{\Delta}, \xi_{\Delta}\right)
$$

This limit does exist because the ratio on the right-hand side is decreasing in $\Delta$, as follows from inequality (A.2). (As before, we set a ratio of determinants equal to zero if the denominator vanishes.) In contrast to (3.6), the determinants in (3.7) involve $J$ itself rather than $J_{\left[\Delta_{n}\right]}$. Now our question is the following: When is it true that $\widehat{c}=\widehat{c}_{*}$ a.e. for $L \otimes \mu$ ?

Unfortunately, we are unable to settle the question above in the same generality as this was done in the lattice case by Shirai and Takahashi (ref. 25, Theorem 6.2). Their argument exploits the symmetry between occupied and empty lattice sites and therefore does not carry over to our continuous setting. (Formally, this is reflected by their condition spec $K \subset]$ 0, 1[, which is never satisfied in the continuous case.) The following theorem gives a partial answer, at least. 
Theorem 3.7. In general, the inequality $\widehat{c} \leqslant \widehat{c}_{*}$ holds $L \otimes \mu$-almost everywhere. If $\widehat{c}_{*}$ is continuous $L \otimes \mu$-a.e. then $\widehat{c}=\widehat{c}_{*} L \otimes \mu$-a.e., i.e., $\widehat{c}_{*}$ is a version of the CPI of $\mu$.

Proposition 3.9 will provide sufficient conditions for the almost-everwhere continuity to hold. First let us comment on the significance of this result.

Remark 3.8. If $\widehat{c}=\widehat{c}_{*}$ a.e. then $\mu$ is a Gibbs measure for a specification $G$ defined in terms of $J$ as follows. For each $\Lambda \in \mathcal{B}_{0}$ and any $\xi \in \mathcal{X}_{\Lambda^{c}}$ satisfying $\operatorname{det} J(\zeta, \zeta)>0$ for all finite $\zeta \subset \xi$, let $G_{\Lambda}(\cdot \mid \xi)$ be defined by inserting $\widehat{c}_{*}$ into (2.10). In fact, $G_{\Lambda}(\cdot \mid \xi)$ is a Gibbs distribution for the Hamiltonian $H_{\Lambda}(\cdot \mid \xi)=-\log \widehat{c}_{*}(\cdot, \xi)$ on $\mathcal{X}_{\Lambda}, \xi \in \mathcal{X}_{\Lambda^{c}}$. (If desired, one can express the Hamiltonian in terms of a many-body potential $\Phi$, but this is not particularly useful.) These Gibbs distributions altogether form a Gibbsian specification $G=\left(G_{\Lambda}\right)_{\Lambda \in \mathcal{B}_{0}}$ in the sense of Preston (ref. 21, pp. 16-17); this has been proved by Glötzl ${ }^{(6)}$ in a general setting, and in ref. 32 for the particular case of DPP's. In particular, if $\widehat{c}_{*}$ is a.e. continuous then the conditional expectations $\mathbb{E}_{\mu}\left(g \mid \mathcal{F}_{\Lambda^{c}}\right)$ in (2.8) have a.e. continuous versions. This is Gibbsianness in the best sense one can expect for a point process.

Conversely, suppose $\mu$ is a Gibbs measure for some Hamiltonian $H$. Then $\widehat{c}^{H}(\alpha, \xi):=\exp \left[-H_{\alpha}\left(\alpha \mid \xi_{\alpha^{c}}\right)\right]$ is a version of its $\mathrm{CPI}^{(17)}$ and will typically satisfy the continuity condition $\widehat{c}^{H}(\alpha, \xi)=\lim _{\Delta \uparrow E} \widehat{c}^{H}\left(\alpha, \xi_{\Delta}\right)$. If one assumes that the function $\widehat{c}=\lim _{n \rightarrow \infty} \widehat{c}_{\Delta_{n}}$ in (3.6) has the same continuity property, one can conclude that $\widehat{c}=\widehat{c}_{*}$ a.e. For, Proposition 4.2 implies that $\lim _{n \rightarrow \infty} \widehat{c}_{\Delta_{n}}\left(\alpha, \xi_{\Delta}\right)=\widehat{c}_{*}\left(\alpha, \xi_{\Delta}\right)$ a.e. for all $\Delta \in \mathcal{B}_{0}$.

The next proposition describes a case in which the condition of almost-everwhere continuity in Theorem 3.7 is satisfied.

Proposition 3.9. Suppose $E=\mathbb{R}^{d}, \lambda$ is Lebesgue measure and, in addition to $(\mathrm{H})$, $\mathbb{R}^{d}$;

(a) $J:=K(I-K)^{-1}$ has a continuous integral kernel $J(x, y), x, y \in$

(b) $J$ has finite range $R<\infty$, in that $J(x, y)=0$ for $|x-y|>R$;

(c) $\mu$ does not percolate, in that $\mu\left(\exists\right.$ infinite cluster of $\left.B_{R}(\cdot)\right)=0$.

Then $\widehat{c}_{*}$ is continuous $L \otimes \mu$-almost everywhere. Specifically,

$$
\widehat{c}_{*}(\alpha, \xi)=\operatorname{det} J\left(\alpha \xi_{W}, \alpha \xi_{W}\right) / \operatorname{det} J\left(\xi_{W}, \xi_{W}\right) 1_{\{\operatorname{diam} W(\alpha, \xi)<\infty\}},
$$


where $W(\alpha, \xi)$ is the union of the clusters of $B_{R}(\alpha \xi)$ hitting $\alpha$, and $\xi_{W}:=$ $\xi_{W(\alpha, \xi)}$ is the restriction of $\xi$ to $W(\alpha, \xi)$.

Under the conditions of this proposition, the Gibbsianness of DPP's has already been derived by the second author in ref. 32 by different methods.

Example 3.10. Typical examples of operators satisfying the assumptions of Proposition 3.9 are obtained by Fourier transforms: Let $\widehat{j} \geqslant 0$ be an integrable function on $\mathbb{R}^{d}$,

$$
j(x):=(2 \pi)^{-d} \int_{\mathbb{R}^{d}} e^{i x \cdot t} \widehat{j}(t) d t
$$

its (inverse) Fourier transform, and $J(x, y):=j(x-y)$. We assume that $j(\cdot) \in L^{1}\left(\mathbb{R}^{d}\right)$. By Bochner's theorem and Young's inequality (ref. 22, p. 29), $J$ then defines a positive bounded operator on $L^{2}\left(\mathbb{R}^{d}\right)$. The associated correlation operator $K:=J(I+J)^{-1}$ is the convolution operator for the function $k$ satisfying $\widehat{k}=\widehat{j} /(1+\widehat{j})$. The validity of hypothesis $(\mathrm{H})$ is evident, and the continuity assumption (a) of Proposition 3.9 also holds by definition. To satisfy the finite range condition (b), let $j:=\varphi \chi_{R}$ or, equivalently, $\widehat{j}=\widehat{\varphi} \star \widehat{\chi}_{R}$, where $0 \leqslant \widehat{\varphi} \in L^{1}\left(\mathbb{R}^{d}\right), \chi_{R}(x)=\prod_{i=1}^{d}\left(1-\left|x_{i}\right| / R\right)^{+}$, and $\star$ denotes convolution. By Corollary 3.5, the non-percolation assumption (c) holds when $d=1$ or $j(0)<z_{c}(d, R)$.

To construct non-translation-invariant examples, let $\psi \geqslant 0$ be any bounded measurable function on $\mathbb{R}^{d}$ and $M_{\psi}$ the associated multiplication operator on $L^{2}\left(\mathbb{R}^{d}, \lambda\right)$. Let $J$ be a finite range operator as above and $L:=J M_{\psi} J . L$ has the kernel $L(x, y)=\int j(x-z) \psi(z) j(z-y) d z$. Obviously, $L(x, y)$ is of finite range because so is $j$, and not translation-invariant except, when $\psi$ is constant almost everywhere.

Let us also comment on the particular case of renewal processes which deserves special interest.

Example 3.11. Let $E=\mathbb{R}$ and $K: f \rightarrow k \star f$ the convolution operator for the function $k(x)=\rho e^{-a|x|}$, where $\rho, a>0$ are such that $\rho<a / 2$. (The last condition means that $\|k\|_{1}<1$, and thus $\|K\|<1$ by Young's inequality.) In the setting of the preceding example, this corresponds to setting $\widehat{k}(t)=2 \rho a /\left(a^{2}+t^{2}\right)$. Hence

$$
\widehat{j}(t)=\frac{\widehat{k}(t)}{1-\widehat{k}(t)}=\frac{2 \rho a}{\sigma^{2}+t^{2}}
$$


with $\sigma^{2}:=a^{2}-2 \rho a$, and therefore $j(x)=(\rho a / \sigma) e^{-\sigma|x|}$. The associated integral kernel can be written in the form

$$
J(x, y):=j(x-y)=u(x \wedge y) v(x \vee y)
$$

with $x \wedge y=\min (x, y), x \vee y=\max (x, y), u(x)=e^{\sigma x}$, and $v(x)=(\rho a / \sigma) e^{-\sigma x}$. Therefore, if $\alpha=\left\{x_{1}, \ldots, x_{n}\right\}$ with $x_{1}<\cdots<x_{n}$ then

$$
\operatorname{det} J(\alpha, \alpha)=u\left(x_{1}\right) v\left(x_{n}\right) \prod_{i=1}^{n-1} d\left(x_{i+1}-x_{i}\right)
$$

with $d\left(x_{i+1}-x_{i}\right)=\operatorname{det}\left(\begin{array}{ll}v\left(x_{i}\right) & v\left(x_{i+1}\right) \\ u\left(x_{i}\right) & u\left(x_{i+1}\right)\end{array}\right)=(2 \rho a / \sigma) \sinh \left(\sigma\left(x_{i+1}-x_{i}\right)\right)$, as is easily verified by induction on $n=|\alpha|$. Together with (3.7) we find that

$$
c_{*}(x, \xi)=d\left(\ell_{x}(\xi)\right) d\left(r_{x}(\xi)\right) / d\left(\ell_{x}(\xi)+r_{x}(\xi)\right)
$$

where $\ell_{x}(\xi)$ and $r_{x}(\xi)$ are the distances from $x$ to the nearest points of $\xi$ on the left, respectively, on the right. This relationship still holds if $d(\cdot)$ is multiplied with an exponential factor to obtain a probability density. That is, we have

$$
c_{*}(x, \xi)=f\left(\ell_{x}(\xi)\right) f\left(r_{x}(\xi)\right) / f\left(\ell_{x}(\xi)+r_{x}(\xi)\right)
$$

for the probability density $f(x)=e^{-a x} d(x)$ on $[0, \infty[$. The right-hand side is the PI of the stationary renewal process $\mu_{f}$ with spacing density $f$, and $\mu_{f}$ is the only translation invariant point process having this PI. As $c_{*}$ is continuous, we conclude from Theorem 3.7 that $\mu_{f}$ is the unique DPP for $K$. In this way we recover a well-known result of $\mathrm{Macchi}^{(13)}$ (see also ref. 4). According to Soshnikov, ${ }^{(28)}$ the densities $f$ of the form above are the only possible spacing distributions of determinantal renewal processes. (We remark that Soshnikov also gave a complete characterization of all kernels $K(x, y)$ on $E=[0, \infty[$, which are such that the associated DPP's have independent spacings (see also Example 6.7 of ref. 24). The previous comments can be extended to this setting.) 


\section{PROOFS}

Let us start with the proof of Theorem 3.1. Its key ingredients are Eq. (2.4) for the Janossy densities of $\mu$, and the determinant inequalities of Lemma A.1 in Appendix. We assume throughout this section that hypothesis $(\mathrm{H})$ is satisfied.

Proof of Theorem 3.1. Fix any $\Lambda \in \mathcal{B}_{0}$. From Eq. (2.4), we know that $\mu_{\Lambda}$ is absolutely continuous relative to $L_{\Lambda}$ with density $\sigma_{\Lambda}(\xi) \propto$ det $J_{[\Lambda]}(\xi, \xi)$. Inequality (A.1) implies that the zero set $\left\{\sigma_{\Lambda}=0\right\}$ is increasing. Remark 2.7 thus tells us that $\mu_{\Lambda}$ satisfies condition $\left(\Sigma_{\lambda_{\Lambda}}\right)$ with PI given by (3.1). This proves the first statement of the theorem.

For the proof of (3.2) let $\xi \subset \eta \in \mathcal{X}_{\Lambda}$ and $\alpha \in \mathcal{X}_{0}$ with $\alpha \subset \Lambda \backslash \eta$. Choosing the kernel function of $J_{[\Lambda]}$ as in Lemma A.3, we get that $J_{[\Lambda]}(\alpha \eta, \alpha \eta)$ is positive definite. Inequality (A.2) thus asserts that

$$
\operatorname{det} J_{[\Lambda]}(\alpha \eta, \alpha \eta) \operatorname{det} J_{[\Lambda]}(\xi, \xi) \leqslant \operatorname{det} J_{[\Lambda]}(\eta, \eta) \operatorname{det} J_{[\Lambda]}(\alpha \xi, \alpha \xi)
$$

and this gives the first inequality in (3.2). In particular, we have $\widehat{c}_{\Lambda}(\alpha, \xi) \leqslant$ $c_{\Lambda}(\alpha, \emptyset)$ for the empty configuration $\emptyset$. But the last quantity equals $\operatorname{det} J_{[\Lambda]}(\alpha, \alpha)$ by definition. An application of inequality (A.1) then gives the last estimate.

To exploit the upper bound in (3.2) we will use the following comparison lemma.

Lemma 4.1. For any $\Lambda \subset \Delta \in \mathcal{B}_{0}, J_{[\Lambda]} \leqslant P_{\Lambda} J_{[\Delta]} P_{\Lambda} \leqslant J_{\Lambda}$ in the operator ordering. In particular,

$$
\operatorname{det} J_{[\Lambda]}(\xi, \xi) \leqslant \operatorname{det} J_{[\Delta]}(\xi, \xi) \leqslant \operatorname{det} J_{\Lambda}(\xi, \xi)
$$

for $L_{\Lambda}$-almost all $\xi \in \mathcal{X}_{\Lambda}$.

Proof. We prove only the inequality $J_{[\Lambda]} \leqslant J_{\Lambda}$, which implies in particular that $P_{\Lambda} J_{[\Delta]} P_{\Lambda} \leqslant P_{\Lambda} J_{\Delta} P_{\Lambda}=J_{\Lambda}$. The proof of the relation $J_{[\Lambda]} \leqslant$ $P_{\Lambda} J_{[\Delta]} P_{\Lambda}$ is similar. Since $J=K(I-K)^{-1}$ and $L(I-L)^{-1}=-I+(I-$ $L)^{-1}$ for any contraction $L$, the inequality $J_{[\Lambda]} \leqslant J_{\Lambda}$ is equivalent to

$$
P_{\Lambda}\left(I-K_{\Lambda}\right)^{-1} P_{\Lambda} \leqslant P_{\Lambda}(I-K)^{-1} P_{\Lambda} .
$$

But this follows from Lemma A.5 as applied to $P=P_{\Lambda}$ and $T=I-K$, because $P_{\Lambda}\left(I-K_{\Lambda}\right)^{-1} P_{\Lambda}=P_{\Lambda}\left(P_{\Lambda}(I-K) P_{\Lambda}\right)^{-1} P_{\Lambda}$. 
To prove the determinantal inequalities (4.1), we apply Lemma A.3 to the positive trace class operators $J_{[\Lambda]}, P_{\Lambda} J_{\Delta} P_{\Lambda}-J_{[\Lambda]}$, and $J_{\Lambda}-P_{\Lambda} J_{\Delta} P_{\Lambda}$. We then see that the kernels of $J_{[\Lambda]}, J_{\Delta}$, and $J_{\Lambda}$ can be chosen in such a way that, for each $\xi \in \mathcal{X}_{\Lambda}, J_{[\Lambda]}(\xi, \xi) \leqslant J_{\Delta}(\xi, \xi) \leqslant J_{\Lambda}(\xi, \xi)$ as operators on $\mathbb{C}^{|\xi|}$. By Lemma A.2, these kernels are indistinguishable from the corresponding kernels obtained by applying Lemma A.3 to $J_{[\Lambda]}, J_{\Delta}$, and $J_{\Lambda}$ directly. For the latter kernels, the same operator inequalities thus hold for $L_{\Lambda}$-almost all $\xi \in \mathcal{X}_{\Lambda}$, at least. To complete the proof it is therefore sufficient to note that the determinant is increasing relative to the operator ordering, (see ref. 1, Corollary III.2.3).

We are now ready for the proofs of Remark 3.2 and Corollaries 3.33.5 .

Proof of Remark 3.2. (a) Let us start with a slightly simpler estimate. Combining the last bound in (3.2) with the second inequality in (4.1) (for $\xi=\{x\}$ ) and using (2.1) and the trace formula (A.6) we find

$$
Z_{\Lambda, \Delta}(\xi) \leqslant \exp \int_{\Lambda} J_{\Lambda}(x, x) \lambda(d x)=\exp \operatorname{Tr} J_{\Lambda}<\infty
$$

because $J_{\Lambda}$ is trace class by $(\mathrm{H})$.

To obtain the sharper bound stated in Remark 3.2(a) we use the next to last inequality in (3.2) and the second inequality in (4.1) (for $\xi=\alpha$ ) to obtain

$$
Z_{\Lambda, \Delta}(\xi) \leqslant \int L_{\Lambda}(d \alpha) \operatorname{det} J_{\Lambda}(\alpha, \alpha)=\operatorname{det}\left(I+J_{\Lambda}\right)
$$

To get the last identity one can use the argument on p. 930 of ref. 28 by combining Lidskii's theorem (ref. 27, p. 50) with the trace formula (A.6). (A similar argument implies that the Janossy densities in (2.4) integrate to 1.) We note that $\operatorname{det}\left(I+J_{\Lambda}\right) \leqslant \exp \operatorname{Tr}\left(J_{\Lambda}\right)$ by (ref. 27, equation (3.6)).

(b) For fixed $\xi$ as stated, $G_{\Lambda, \Delta}(\cdot \mid \xi)$-considered as a measure on $\mathcal{X}_{\Lambda}$-is defined by an $L_{\Lambda}$-density of the form

$$
\sigma_{\Lambda, \Delta}^{\xi}(\alpha) \propto \widehat{c}_{\Delta}(\alpha, \xi)=\operatorname{det} J_{[\Delta]}(\alpha \xi, \alpha \xi) / \operatorname{det} J_{[\Delta]}(\xi, \xi), \quad \alpha \in \mathcal{X}_{\Lambda} .
$$

In view of the identity (A.3), the last expression is equal to $\operatorname{det} J_{\Lambda, \Delta}^{\xi}(\alpha, \alpha)$. Comparing this observation with (2.4) we see that $G_{\Lambda, \Delta}(\cdot \mid \xi)$ is a determinantal process with interaction kernel $J_{\Lambda, \Delta}^{\xi}$, defining a positive Hermitian operator on $L^{2}\left(\Lambda, \lambda_{\Lambda}\right)$. The associated correlation operator is $K_{\Lambda, \Delta}^{\xi}:=$ $J_{\Lambda, \Delta}^{\xi}\left(I+J_{\Lambda, \Delta}^{\xi}\right)^{-1}$. 
Proof of Corollary 3.3. In view of Eq. (3.3),

$$
G_{\Lambda, \Delta}(f \mid \xi) / G_{\Lambda, \Delta}\left(N_{\Lambda}=0 \mid \xi\right)=\int L_{\Lambda}(d \alpha) f(\alpha) \widehat{c}_{\Delta}(\alpha, \xi)
$$

for $\mu_{\Delta}$-almost all $\xi \in \mathcal{X}_{\Delta \backslash \Lambda}$. Since $f \geqslant 0$, Theorem 3.1(b) together with Eq. (2.6) implies that the integrand on the right-hand side is a decreasing function of $\xi$. In particular, for $f \equiv 1$ we find that $G_{\Lambda, \Delta}\left(N_{\Lambda}=0 \mid \cdot\right)$ is increasing. To prove (3.4) we note that

$$
\begin{aligned}
& \mu\left(N_{\Lambda}=0 \mid N_{\Delta_{2} \backslash \Lambda}=0\right)=G_{\Lambda, \Delta_{2}}\left(N_{\Lambda}=0 \mid \emptyset\right) \\
& \quad \leqslant \mathbb{E}_{\mu}\left(G_{\Lambda, \Delta_{2}}\left(N_{\Lambda}=0 \mid \cdot\right) \mid \mathcal{F}_{\Delta_{1} \backslash \Lambda}\right)(\emptyset) \\
& \quad=\mu\left(N_{\Lambda}=0 \mid \mathcal{F}_{\Delta_{1} \backslash \Lambda}\right)(\emptyset)=\mu\left(N_{\Lambda}=0 \mid N_{\Delta_{1} \backslash \Lambda}=0\right)
\end{aligned}
$$

because $G_{\Lambda, \Delta_{2}}\left(N_{\Lambda}=0 \mid \emptyset\right) \leqslant G_{\Lambda, \Delta_{2}}\left(N_{\Lambda}=0 \mid \cdot\right)$. The final inequality follows by setting $\Delta_{1}=\Lambda$.

Proof of Corollary 3.4. We show first that $\mu_{\Lambda} \preceq \pi_{\Lambda}^{J}$ for any $\Lambda \in \mathcal{B}_{0}$. Consider the PI $c_{\Lambda}$ of $\mu_{\Lambda}$. Combining (3.2) (for $\alpha=\{x\}$ ) with (4.1) (for $\xi=\{x\})$ we find that $c_{\Lambda}(x, \xi) \leqslant J_{\Lambda}(x, x)$ for all $x \in \Lambda$ and $\xi \in \mathcal{X}_{\Lambda}$. On the other hand, it is well known and easy to check that the Poisson point process $\pi_{\Lambda}^{J}$ has the PI $c_{\Lambda}^{J}(x, \xi)=J(x, x)$ on $\Lambda$ (cf. refs. 15 and 17). Moreover, since $J(x, x)=J_{\Lambda}(x, x)$ for $\lambda$-a.a. $x \in \Lambda$ by the construction of $J(x, x)$ in Lemma A.4, we may also regard $J_{\Lambda}(x, x)$ as the PI of $\pi_{\Lambda}^{J}$. Thus, we can conclude that the PI of $\mu_{\Lambda}$ at a configuration $\xi \in \mathcal{X}_{\Lambda}$ is not larger than the PI of $\pi_{\Lambda}^{J}$ evaluated at any larger configuration $\eta \in \mathcal{X}_{\Lambda}$, as long as $x \notin$ $\eta$. This, however, is precisely the hypothesis of the point-process counterpart of the well-known FKG-Holley-Preston inequality for lattice systems. This continuous counterpart was obtained first by Preston, ${ }^{(20)}$ an alternative simplified proof can be found in (ref. 5, Theorem 1.1). It asserts that, under the above condition on the PI's, $\mu_{\Lambda} \preceq \pi_{\Lambda}^{J}$, as required.

To get rid of the locality restriction, we argue as follows. For any compact $\Lambda$, a celebrated theorem of Strassen ${ }^{(31)}$ provides us with a probability measure $m_{\Lambda}$ on $\mathcal{X} \times \mathcal{X}$ having marginals $\mu_{\Lambda}$ resp. $\pi_{\Lambda}^{J}$ and being supported on the set $D:=\{(\xi, \eta) \in \mathcal{X} \times \mathcal{X}: \xi \subset \eta\}$. Note that $D$ is closed when $\mathcal{X} \times \mathcal{X}$ is equipped with the product of the vague topology on $\mathcal{X}$. By a standard compactness criterion for point processes (ref. 4, Proposition 9.1.V), the measures $m_{\Lambda}$ admit a weak limiting measure $m$ as $\Lambda$ increases to $E$. By construction, $m$ has marginals $\mu$ resp. $\pi^{J}$, and $m(D)=1$ because $D$ is closed. This implies that $\mu \preceq \pi^{J}$. 
Proof of Corollary 3.5. By assumption, there exists some $z<z_{c}$ such that $J(x, x) \leqslant z$ for all $x$ outside of some compact region. Therefore, $\pi^{J}$-almost surely there exists no infinite cluster outside of this region, and therefore no infinite cluster anywhere. As the existence of an infinite cluster is an increasing event, the absence of percolation carries over to $\mu$, by Corollary 3.4 .

Next we turn to the proof of Theorem 3.6. We will use a martingale argument to derive property $\left(\Sigma_{\lambda}\right)$ from the local property $\left(\Sigma_{\lambda_{\Lambda}}\right)$ of Theorem 3.1.

Proof of Theorem 3.6. We only need to show that $\mu$ satisfies condition $\left(\Sigma_{\lambda}\right)$ with CPI (3.6); the remaining assertions then follow from Theorem 3.1 and Remark 2.5(c). Let $\left(\Delta_{n}\right)$ be any increasing sequence in $\mathcal{B}_{0}$ exhausting $E, \Lambda \in \mathcal{B}_{0}$ a fixed set, and $n$ so large that $\Lambda \subset \Delta_{n}$. Consider the product space $\mathcal{X}_{\Lambda} \times \mathcal{X}$, equipped with the probability measure $v_{\Lambda}:=$ $\pi_{\Lambda}^{1} \otimes \mu$ and the $\sigma$-algebras $\mathcal{G}_{n}=\left(\mathcal{F} \mid \mathcal{X}_{\Lambda}\right) \otimes \mathcal{F}_{\Delta_{n}}$. Now, Theorem 3.1 asserts that, on $\mathcal{G}_{n}$, the restriction of $\widehat{C}_{\mu}$ to $\mathcal{X}_{\Lambda} \times \mathcal{X}$ is absolutely continuous with respect to $v_{\Lambda}$ with Radon-Nikodym density $R_{n}:=e^{\lambda(\Lambda)} \widehat{c}_{\Delta_{n}}$. The sequence $\left(R_{n}\right)$ is therefore a non-negative martingale relative to $\nu_{\Lambda}$. By (3.2) and (4.1), $R_{n}$ satisfies the bound

$$
R_{n}(\alpha, \xi) \leqslant e^{\lambda(\Lambda)} \operatorname{det} J_{\left[\Delta_{n}\right]}(\alpha, \alpha) \leqslant S(\alpha):=e^{\lambda(\Lambda)} \operatorname{det} J_{\Lambda}(\alpha, \alpha)
$$

for $v_{\Lambda}$-almost all $(\alpha, \xi) \in \mathcal{X}_{\Lambda} \times \mathcal{X}$. As we have seen in the proof of Remark 3.2(a), $S$ is integrable relative to $\pi_{\Lambda}^{1}$. As a consequence, $\left(R_{n}\right)$ converges $v_{\Lambda}$-almost surely and in $L^{1}\left(v_{\Lambda}\right)$-norm to a limit $R$. By the norm-convergence, $R$ is a Radon-Nikodym density of $\widehat{C}_{\mu}$ relative to $v_{\Lambda}$ on the limiting $\sigma$-algebra $\sigma\left(\bigcup_{n} \mathcal{G}_{n}\right)=\left(\left.\mathcal{F}\right|_{\mathcal{X}_{\Lambda}}\right) \otimes \mathcal{F}$. Finally we replace $\pi_{\Lambda}^{1}$ with $L_{\Lambda}$ by dropping the constant factor $e^{\lambda(\Lambda)}$, and use the fact that $\mathcal{X}_{\Lambda} \uparrow \mathcal{X}_{0}$ as $\Lambda \uparrow E$. We then get the desired result that $\widehat{C}_{\mu} \ll L \otimes \mu$ with density (3.6).

Our proof of Theorem 3.7 will be based on the following convergence result. As before, we say that a kernel function $t(x, y)$ of an operator $T$ is positive definite almost everywhere if the matrix $t(\alpha, \alpha)$ is positive definite for $L$-almost all $\alpha \in \mathcal{X}_{0}$.

Proposition 4.2. Given any sequence $\left(\Delta_{n}\right)$ in $\mathcal{B}_{0}$ increasing to $E$, there exist integral kernels $J_{[n]}(x, y)$ for $J_{[n]}:=J_{\left[\Delta_{n}\right]}$ such that the following holds: 
(a) Each $J_{[n]}(x, y)$ is positive definite a.e. and satisfies the trace formula

$$
\operatorname{Tr}\left(P_{\Lambda} J_{[n]} P_{\Lambda}\right)=\int_{\Lambda} J_{[n]}(x, x) \lambda(d x)
$$

for all $\Lambda \subset \Delta_{n}$.

(b) The limit

$$
J(x, y)=\lim _{n \rightarrow \infty} J_{[n]}(x, y)
$$

exists for all $x, y \in E \backslash \mathcal{N}$, where $\mathcal{N} \in \mathcal{B}$ is a $\lambda$-nullset. Moreover, this limit defines an integral kernel of $J$, is positive definite a.e., and satisfies the trace formula (3.5).

Note that, by Lemma A.2, the kernels $J_{[n]}(x, y)$ and $J(x, y)$ constructed here are indistinguishable from those obtained directly from Lemmas A.3 and A.4.

Proof. In view of hypothesis $(\mathrm{H})$ and Lemma A.4, $K$ admits an integral kernel $K(x, y)$ with the properties (a)-(c) of that lemma. In particular, $K(x, y)$ is positive definite a.e., and there exists a $\lambda$-nullset $\mathcal{N} \in \mathcal{B}$ such that $k_{x}:=K(\cdot, x) \in L^{2}(E, \lambda)$, whenever $x \notin \mathcal{N}$. For each $n$ we choose the integral kernel $K_{n}(x, y):=1_{\Delta_{n}}(x) K(x, y) 1_{\Delta_{n}}(y), x, y \in \Delta_{n}$, of $K_{n}:=K_{\Delta_{n}}$. Also, for each power $l \geqslant 2$ we set $K^{l}(x, y):=\left(k_{x}, K^{l-2} k_{y}\right)$ and $K_{n}^{l}(x, y):=$ $\left(k_{x}, P_{n}\left(K_{n}\right)^{l-2} k_{y}\right)$, where $(\cdot, \cdot)$ is the inner product in $L^{2}(E, \lambda)$ and $P_{n}:=$ $P_{\Delta_{n}}$. These are well defined for $x, y \notin \mathcal{N}$, and define versions of the integral kernels of $K^{l}$ resp. $\left(K_{n}\right)^{l}$.

Now, since $\left\|K_{n}\right\| \leqslant\|K\|<1$, we can write $J_{[n]}=\sum_{l \geqslant 1}\left(K_{n}\right)^{l}$ and $J=$ $\sum_{l \geqslant 1} K^{l}$; these series converge in operator norm, and the convergence is uniform in $n$. In particular, this implies the absolute convergence of the series $\sum_{l \geqslant 1} K_{n}^{l}(x, y)$ and $\sum_{l \geqslant 1} K^{l}(x, y)$ for $x, y \notin \mathcal{N}$, again uniformly in $n$. Obviously, these series define integral kernels of $J_{[n]}$ and $J$, respectively. They are positive definite a.e. because $K(x, y)$ is.

To prove the trace formula $(3.5)$ we introduce the partial sum $J^{(m)}=$ $\sum_{l=1}^{m} K^{l}$. Obviously, as $m \rightarrow \infty$, the sequence $P_{\Lambda} J^{(m)} P_{\Lambda}$ increases and converges in operator norm to $J_{\Lambda}$, which is of trace class. Using (ref. 27, Theorem 2.16), we conclude that $P_{\Lambda} J^{(m)} P_{\Lambda} \rightarrow J_{\Lambda}$ in trace norm as $m \rightarrow \infty$. Hence

$$
\operatorname{Tr}\left(J_{\Lambda}\right)=\lim _{m \rightarrow \infty} \sum_{l=1}^{m} \operatorname{Tr}\left(P_{\Lambda} K^{l} P_{\Lambda}\right) .
$$


Inserting the trace formulas of Lemma A.4(c) and taking the limit $m \rightarrow \infty$ we arrive at (3.5). Formula (4.2) follows in the same way by considering $J_{[n]}$ resp. $K_{n}$ instead of $J$ resp. $K$.

It remains to show that $J_{[n]}(x, y) \rightarrow J(x, y)$ when $x, y \notin \mathcal{N}$. By the uniform absolute convergence of these series, it is enough to check that $K_{n}^{l}(x, y) \rightarrow K^{l}(x, y)$ for all $l \in \mathbb{N}$. The case $l=1$ is trivial. For $l=2$ we have $K_{n}^{2}(x, y)=\left(k_{x}, P_{n} k_{y}\right)$ when $\Delta_{n} \ni x, y$, and this converges to $\left(k_{x}, k_{y}\right)=$ $K^{2}(x, y)$. In the case $l \geqslant 3$ we note that $K_{n}$ converges strongly to $K$ as $n \rightarrow \infty$. Since $\left\|K_{n}\right\| \leqslant 1$, it follows that $\left(K_{n}\right)^{l-2}$ converges strongly to $K^{l-2}$, whence $K_{n}^{l}(x, y)=\left(k_{x},\left(K_{n}\right)^{l-2} k_{y}\right)$ converges to $\left(k_{x}, K^{l-2} k_{y}\right)=$ $K^{l}(x, y)$. This completes the proof.

\section{Proof of Theorem 3.7. Consider the CPI}

$$
\widehat{c}(\alpha, \xi)=\lim _{n \rightarrow \infty} \widehat{c}_{\Delta_{n}}\left(\alpha, \xi_{\Delta_{n}}\right)
$$

that occurs in (3.6). We know that this limit exists for $L \otimes \mu$-almost all $(\alpha, \xi) \in \mathcal{X}_{0} \times \mathcal{X}$. In fact, we may define $\widehat{c}_{\Delta_{n}}\left(\alpha, \xi_{\Delta_{n}}\right)$ in terms of the integral kernels $J_{[n]}$ of Proposition 4.2 by setting $\widehat{c}_{\Delta_{n}}\left(\alpha, \xi_{\Delta_{n}}\right)=\operatorname{det} J_{[n]}\left(\alpha \xi_{\Delta_{n}}, \alpha \xi_{\Delta_{n}}\right) /$ $\operatorname{det} J_{[n]}\left(\xi_{\Delta_{n}}, \xi_{\Delta_{n}}\right)$. On the other hand, for each $\Lambda \in \mathcal{B}_{0}$ we conclude from (3.2) that $\widehat{c}_{\Delta_{n}}\left(\alpha, \xi_{\Delta_{n}}\right) \leqslant \widehat{c}_{\Delta_{n}}\left(\alpha, \xi_{\Lambda}\right)$ for $L \otimes \mu$-a.a. $(\alpha, \xi)$ as soon as $\Delta_{n} \supset \Lambda$. Hence

$$
\widehat{c}(\alpha, \xi) \leqslant \liminf _{n \rightarrow \infty} \widehat{c}_{\Delta_{n}}\left(\alpha, \xi_{\Lambda}\right)
$$

Now, Proposition 4.2 implies that for $L \otimes L_{\Lambda}$-a.a. $\left(\alpha, \xi_{\Lambda}\right)$

$$
\widehat{c}_{\Delta_{n}}\left(\alpha, \xi_{\Lambda}\right) \rightarrow \widehat{c}_{*}\left(\alpha, \xi_{\Lambda}\right)=\operatorname{det} J\left(\alpha \xi_{\Lambda}, \alpha \xi_{\Lambda}\right) / \operatorname{det} J\left(\xi_{\Lambda}, \xi_{\Lambda}\right)
$$

as $n \rightarrow \infty$, provided that $\operatorname{det} J\left(\xi_{\Lambda}, \xi_{\Lambda}\right)>0$. In view of (2.4) and (4.1), the last proviso holds true for $\mu$-almost all $\xi$. Hence $\widehat{c}(\alpha, \xi) \leqslant \widehat{c}_{*}\left(\alpha, \xi_{\Lambda}\right)$ for $L \otimes$ $\mu$-almost all $(\alpha, \xi)$. As we have noticed after (3.7), $\widehat{c}_{*}\left(\alpha, \xi_{\Lambda}\right)$ is a decreasing function of $\Lambda$, and its limit is equal to $\widehat{c}_{*}(\alpha, \xi)$ by definition. This proves that $\widehat{c} \leqslant \widehat{c}_{*}$ almost everywhere.

Suppose next that $\widehat{c}_{*}$ is continuous $L \otimes \mu$-everywhere. Consider the DPP's $\mu^{J_{\Delta}}$ for the interaction operators $J_{\Delta}=P_{\Delta} J P_{\Delta}$, and thus for the correlation operators $K_{[\Delta]}=J_{\Delta}\left(I+J_{\Delta}\right)^{-1}, \Lambda \subset \Delta \in \mathcal{B}_{0}$. According to Theorem 6.17 of ref. 24, or Lemma 4.3 of ref. 32 in the case of a discontinuous kernel of $J, \mu^{J_{\Delta}}$ converges weakly to $\mu$ as $\Delta$ increases to $E$. Let $f$ be any bounded continuous function on $\mathcal{X}_{0} \times \mathcal{X}$ such that $f(\alpha, \xi)=0$ unless 
$\alpha \subset \Lambda$ and $\left|\alpha \xi_{\Lambda}\right| \leqslant k$ for some number $k$. The portmanteau theorem (ref. 4, Proposition A2.3.V) then implies that

$$
\int f \widehat{c}_{*} d\left(L_{\Lambda} \otimes \mu\right)=\lim _{\Delta \uparrow E} \int f \widehat{c}_{*} d\left(L_{\Lambda} \otimes \mu^{J_{\Delta}}\right) .
$$

But $\mu^{J_{\Delta}}$ is supported on $\mathcal{X}_{\Delta}$, and for almost every $\alpha \in \mathcal{X}_{\Lambda}$ and $\xi \in \mathcal{X}_{\Delta}$ we have $\widehat{c}_{*}(\alpha, \xi)=\widehat{c}_{*}^{J_{\Delta}}(\alpha, \xi):=\operatorname{det} J_{\Delta}(\alpha \xi, \alpha \xi) / \operatorname{det} J_{\Delta}(\xi, \xi)$. By Remark 2.7, $\widehat{c}_{*}^{J_{\Delta}}$ is a CPI of $\mu^{J_{\Delta}}$. Equation (2.7) thus shows that

$$
\int f \widehat{c}_{*} d\left(L_{\Lambda} \otimes \mu^{J_{\Delta}}\right)=\int \mu^{J_{\Delta}}(d \xi) \sum_{\alpha \in \mathcal{X}_{0}: \alpha \subset \xi} f(\alpha, \xi \backslash \alpha) .
$$

The integrand on the right-hand side is still a bounded continuous function of $\xi$. Letting $\Delta \uparrow E$ we thus find that

$$
\int f \widehat{c}_{*} d\left(L_{\Lambda} \otimes \mu\right)=\int f d \widehat{C}_{\mu} \mid \mathcal{X}_{\Lambda} \times \mathcal{X}
$$

As $f, k$ and $\Lambda$ were arbitrarily chosen, it follows that $\widehat{c}_{*}$ is a CPI of $\mu$.

Finally we prove Proposition 3.9 on the a.e. continuity of $\widehat{c}_{*}$.

Proof of Proposition 3.9. Fix any $\Lambda \in \mathcal{B}_{0}, \alpha \in \mathcal{X}_{\Lambda}$, and consider the function $\widehat{c}_{*}(\alpha, \cdot)$. For $\xi \in \mathcal{X}$ let $W(\alpha, \xi)$ be the union of all clusters of $B_{R}(\alpha \xi)$ hitting $\alpha$, and write $\xi_{W}=\xi_{W(\alpha, \xi)}$. By the finite range assumption, $J(x, y)=0$ whenever $x \in \alpha \xi_{W}$ and $y \in \xi \backslash \xi_{W}$. Hence, if $W(\alpha, \xi)$ is finite then, for any local $\Delta \supset W(\alpha, \xi)$, the matrix $J\left(\alpha \xi_{\Delta}, \alpha \xi_{\Delta}\right)$ consists of the two diagonal blocks $J\left(\alpha \xi_{W}, \alpha \xi_{W}\right)$ and $J\left(\xi_{\Delta \backslash W}, \xi_{\Delta \backslash W}\right)$. Thus

$$
\operatorname{det} J\left(\alpha \xi_{\Delta}, \alpha \xi_{\Delta}\right)=\operatorname{det} J\left(\alpha \xi_{W}, \alpha \xi_{W}\right) \operatorname{det} J\left(\xi_{\Delta \backslash W}, \xi_{\Delta \backslash W}\right)
$$

Dividing this by the analogous equation for $\alpha=\emptyset$ we arrive at (3.8). By the continuity of the kernel $J$, it follows that $\widehat{c}_{*}(\alpha, \cdot)$ is continuous on the open set of configurations having no infinite cluster, which has full measure by assumption.

\section{APPENDIX A}

Here we collect some general facts on positive matrices and operators. Some of them are standard and listed here for ease of reference. (We include their simple proofs for the convenience of the readers.) Some others are more subtle and do not seem to appear in the literature, at least 
not in the form we need. We start with a classical lemma on determinants of finite matrices. The inequalities (A.1) and (A.2) are a key ingredient of our arguments; (A.1) is known as Fischer's inequality. To state the lemma we consider a finite index set $\Gamma$ and any complex matrix $A=\left(a_{i j}\right)_{i, j \in \Gamma}$. For any two subsets $\alpha, \beta \subset \Gamma$ we let $A_{\alpha, \beta}=\left(a_{i j}\right)_{i \in \alpha, j \in \beta}$ be the associated submatrix.

Lemma A.1. If $A$ is Hermitian and positive definite then

$$
\operatorname{det} A \leqslant \operatorname{det} A_{\alpha, \alpha} \operatorname{det} A_{\beta, \beta},
$$

when $\Gamma=\alpha \cup \beta$ is partitioned into two nontrivial subsets, and

$$
\operatorname{det} A \operatorname{det} A_{\beta, \beta} \leqslant \operatorname{det} A_{\alpha \cup \beta, \alpha \cup \beta} \operatorname{det} A_{\beta \cup \gamma, \beta \cup \gamma} \text {, }
$$

when $\Gamma=\alpha \cup \beta \cup \gamma$ is partitioned into three nontrivial subsets. For $\Gamma=\alpha \cup$ $\beta$ and general $A$ with invertible $A_{\beta, \beta}$ we have

$$
\operatorname{det} A / \operatorname{det} A_{\beta, \beta}=\operatorname{det}\left(a_{k l}^{\beta}\right)_{k, l \in \alpha}
$$

with $a_{k l}^{\beta}=\operatorname{det} A_{\beta \cup\{k\}, \beta \cup\{l\}} / \operatorname{det} A_{\beta, \beta}$.

Proof. If $\Gamma=\alpha \cup \beta$ and $A_{\beta, \beta}$ is invertible we can write

$$
A=\left(\begin{array}{cc}
A_{\alpha, \alpha} & A_{\alpha, \beta} \\
A_{\beta, \alpha} & A_{\beta, \beta}
\end{array}\right)=\left(\begin{array}{cc}
A_{\alpha, \alpha}-A_{\alpha, \beta} A_{\beta, \beta}^{-1} A_{\beta, \alpha} & A_{\alpha, \beta} A_{\beta, \beta}^{-1} \\
0 & I
\end{array}\right)\left(\begin{array}{cc}
I & 0 \\
A_{\beta, \alpha} & A_{\beta, \beta}
\end{array}\right),
$$

where $I$ is the identity matrix. Hence

$$
\operatorname{det} A / \operatorname{det} A_{\beta, \beta}=\operatorname{det}\left(A_{\alpha, \alpha}-A_{\alpha, \beta} A_{\beta, \beta}^{-1} A_{\beta, \alpha}\right) \text {. }
$$

Now, if $A$ is Hermitian and positive definite then $A_{\alpha, \beta} A_{\beta, \beta}^{-1} A_{\beta, \alpha} \geqslant 0$, and the determinant is monotone with respect to the operator ordering. This implies (A.1) for invertible $A_{\beta, \beta}$. For general $A_{\beta, \beta}$ one can approximate $A_{\beta, \beta}$ by invertible matrices. To prove (A.2) one can assume that det $A_{\beta, \beta}>$ 0 and then divide both sides by $\operatorname{det}^{2} A_{\beta, \beta}$. In view of (A.4), (A.2) then takes the form

$$
\begin{aligned}
& \operatorname{det}\left(A_{\alpha \cup \gamma, \alpha \cup \gamma}-A_{\alpha \cup \gamma, \beta} A_{\beta, \beta}^{-1} A_{\beta, \alpha \cup \gamma}\right) \\
& \quad \leqslant \operatorname{det}\left(A_{\alpha, \alpha}-A_{\alpha, \beta} A_{\beta, \beta}^{-1} A_{\beta, \alpha}\right) \operatorname{det}\left(A_{\gamma, \gamma}-A_{\gamma, \beta} A_{\beta, \beta}^{-1} A_{\beta, \gamma}\right),
\end{aligned}
$$


which follows from (A.1). To verify (A.3) we have to show that the righthand sides of (A.4) and (A.3) are identical. However, applying (A.4) to the matrix $A_{\alpha \cup\{k\}, \alpha \cup\{l\}}$ it is easily seen that $a_{k l}^{\beta}$ is precisely the $k l$-entry of the matrix on the right-hand side of (A.4).

The second subject of this appendix is the proper choice of integral kernels. Note that we need to evaluate such integral kernels at the points of configurations in $E$. This leads us to the following concept. Let us say that two functions $t_{1}, t_{2}: E^{2} \rightarrow \mathbb{C}$ are indistinguishable ( $L$-almost everywhere) if

$$
L\left(\alpha \in \mathcal{X}_{0}: t_{1}(x, y) \neq t_{2}(x, y) \text { for some } x, y \in \alpha\right)=0
$$

The lemma below shows the significance of trace formulas for indistinguishability. As before, we write $T_{\Lambda}=P_{\Lambda} T P_{\Lambda}$.

Lemma A.2. Let $T$ be a local trace class integral operator on $L^{2}(E, \lambda)$ and $t_{1}, t_{2}$ be two integral kernels of $T$ satisfying

$$
\operatorname{Tr} T_{\Lambda}=\int_{\Lambda} t_{i}(x, x) \lambda(d x)
$$

for all $\Lambda \in \mathcal{B}_{0}, i=1,2$. Then $t_{1}$ and $t_{2}$ are indistinguishable.

Proof. Since both $t_{1}$ and $t_{2}$ are integral kernels of $T$, we have $t_{1}=t_{2}$ almost everywhere with respect to $\lambda^{\otimes 2}$. On the other hand, since the local trace formula holds for each $\Lambda \in \mathcal{B}_{0}$, it follows that $t_{1}=t_{2}$ almost everywhere also with respect to $\bar{\lambda}:=\lambda(x:(x, x) \in \cdot)$. So, the set $\mathcal{N}=\left\{t_{1} \neq t_{2}\right\}$ is a nullset for both $\lambda^{\otimes 2}$ and $\bar{\lambda}$. This proves the lemma because

$$
\widehat{\mathcal{N}}:=\left\{\alpha \in \mathcal{X}_{0}:(x, y) \in \mathcal{N} \text { for some } x, y \in \alpha\right\}
$$

is then a nullset for $L$.

As for the existence of suitable integral kernels, we consider first the case of trace class operators. (For $E=\mathbb{R}^{d}$, the trace formula (A.6) appears also in (ref. 28, Lemma 2).) Recall that a function $t: E \times E \rightarrow \mathbb{C}$ is called positive definite if for any $n \in \mathbb{N},\left\{c_{i}\right\}_{i=1}^{n} \subset \mathbb{C}$ and $\left\{x_{i}\right\}_{i=1}^{n} \subset E$,

$$
\sum_{i, j=1}^{n} \bar{c}_{i} t\left(x_{i}, x_{j}\right) c_{j} \geqslant 0 .
$$


Lemma A.3. Let $T \geqslant 0$ be any trace class operator on $L^{2}(E, \lambda)$. Then $T$ admits a positive definite integral kernel $t(x, y)$ satisfying

$$
\operatorname{Tr}\left(P_{\Lambda} T^{k} P_{\Lambda}\right)=\int_{E^{k}} 1_{\Lambda}\left(x_{1}\right) t\left(x_{1}, x_{2}\right) \cdots t\left(x_{k}, x_{1}\right) \lambda\left(d x_{1}\right) \cdots \lambda\left(d x_{k}\right)
$$

for all $k \geqslant 1$ and $\Lambda \in \mathcal{B}$.

Proof. Since $T$ is positive and of trace class, there exists a HilbertSchmidt operator $S$ such that $T=S^{*} S$. Let $s(x, y)$ be an integral kernel function of $S$ and $s^{*}(x, y):=\overline{s(y, x)}$ the associated kernel of $S^{*}$. The Hilbert-Schmidt norm of $S$ satisfies $\|S\|_{2}^{2}=\int_{E^{2}}|s(x, y)|^{2} \lambda(d x) \lambda(d y)<\infty$. There exists therefore a $\lambda$-nullset $\mathcal{N} \subset E$ such that $s(\cdot, y) \in L^{2}(E, \lambda)$ for $y \notin \mathcal{N}$. Define $t(x, y)=\int s^{*}(x, z) s(z, y) \lambda(d z)$ if $x, y \notin \mathcal{N}$ and $t(x, y)=0$ otherwise. Then $t$ is an integral kernel of $T$, and for $\left\{c_{i}\right\}_{i=1}^{n} \subset \mathbb{C}$ and $\left\{x_{i}\right\}_{i=1}^{n} \subset$ $E$ one has

$$
\sum_{i, j=1}^{n} \bar{c}_{i} t\left(x_{i}, x_{j}\right) c_{j}=\int\left|\sum_{j=1}^{n} c_{j} s\left(z, x_{j}\right)\right|^{2} \lambda(d z) \geqslant 0
$$

On the other hand,

$$
\operatorname{Tr} T=\operatorname{Tr} S^{*} S=\|S\|_{2}^{2}=\int_{E^{2}}|s(x, y)|^{2} \lambda(d x) \lambda(d y)=\int_{E} t(x, x) \lambda(d x),
$$

proving (A.6) for $k=1$ and $\Lambda=E$. To check the case $\Lambda \subset E$, it is sufficient to note that $S P_{\Lambda}$ admits the kernel $s(x, y) 1_{\Lambda}(y)$. The case of powers $T^{k}$ with $k>1$ follows similarly because $T^{k}=\left(S^{(k)}\right)^{*} S^{(k)}$ with $S^{(k)}=S^{*} S \cdots S^{\sharp}$, where $S^{\sharp}=S$ if $k$ is even and $S^{\sharp}=S^{*}$ if $k$ is odd.

Next we need to extend Lemma A.3 to integral operators which are only local trace class.

Lemma A.4. Let $T \geqslant 0$ be a bounded local trace class integral operator on $L^{2}(E, \lambda)$. Then one can choose its integral kernel $t(x, y)$ such that the following properties hold:

(a) $t(\alpha, \alpha) \geqslant 0$ for $L$-a.a. $\alpha \in \mathcal{X}_{0}$;

(b) $t(x, y)$ is a Carleman kernel, i.e., $t_{x}:=t(\cdot, x) \in L^{2}(E, \lambda)$ for $\lambda$-a.a. $x \in E$; 
(c) For any $\Lambda \in \mathcal{B}_{0}, \operatorname{Tr}\left(T_{\Lambda}\right)=\int_{\Lambda} t(x, x) \lambda(d x)$ and

$$
\operatorname{Tr}\left(P_{\Lambda} T^{k} P_{\Lambda}\right)=\int_{\Lambda}\left(t_{x}, T^{k-2} t_{x}\right) \lambda(d x)
$$

for $k \geqslant 2$, where $(\cdot, \cdot)$ stands for the inner product in $L^{2}(E, \lambda)$.

Proof. For each $\Delta \in \mathcal{B}_{0}$ we choose an integral kernel $t_{\Delta}$ of $T_{\Delta}$ according to Lemma A.3. For any $\Lambda \subset \Delta, 1_{\Lambda}(x) t_{\Delta}(x, y) 1_{\Lambda}(y)$ is also an integral kernel of $T_{\Lambda}$, which satisfies the assumptions of Lemma A.2. As in the proof of that lemma, we thus find a set $\mathcal{N}_{\Lambda, \Delta}$, which is a nullset for both $\lambda^{\otimes 2}$ and $\bar{\lambda}$ such that $t_{\Lambda}=t_{\Delta}$ on $\Lambda^{2} \backslash \mathcal{N}_{\Lambda, \Delta}$.

Now let $\left(\Delta_{n}\right)_{n \geqslant 1}$ be a sequence in $\mathcal{B}_{0}$ increasing to $E$, and consider the set $\mathcal{N}=\bigcup_{n \geqslant 1} \mathcal{N}_{\Delta_{n}, \Delta_{n+1}}$. For $(x, y) \notin \mathcal{N}$ we let $t(x, y)$ be the common value of all $t_{\Delta_{n}}(x, y)$ with $\Delta_{n} \ni x, y$, and define $t=0$ on $\mathcal{N}$. Since $T$ is supposed to admit an integral kernel $\tilde{t}$, it is then clear that

$$
\int_{E^{2}} \overline{f(x)}[t(x, y)-\widetilde{t}(x, y)] g(y) \lambda(d x) \lambda(d y)=0
$$

whenever $f, g \in L^{2}(E, \lambda)$ vanish outside some $\Delta_{n}$, and hence that $t=\tilde{t}$ $\lambda^{\otimes 2}$-a.e., meaning that $t$ is also a kernel for $T$. By construction, $t$ is indistinguishable from $t_{\Delta_{n}}$ under $L_{\Delta_{n}}$, and $t_{\Delta_{n}}$ is positive definite. This yields assertion (a).

To prove (b) and (c) we proceed as follows. Let $T_{n}:=T_{\Delta_{n}}$ for brevity. Since $T$ is bounded, $P_{\Lambda} T_{n}^{k} P_{\Lambda}$ converges strongly to $P_{\Lambda} T^{k} P_{\Lambda}$ as $n \rightarrow \infty$. In particular, it converges weakly. Since also both $P_{\Lambda} T_{n}^{k} P_{\Lambda}$ and $P_{\Lambda} T^{k} P_{\Lambda}$ are bounded from above by the trace class operator $\|T\|^{k-1} T_{\Lambda}$, it follows that the sequence $\left(P_{\Lambda} T_{n}^{k} P_{\Lambda}\right)_{n \geqslant 1}$ converges to $P_{\Lambda} T^{k} P_{\Lambda}$ in trace norm (ref. 27, Theorem 2.16). Hence

$$
\operatorname{Tr}\left(P_{\Lambda} T^{k} P_{\Lambda}\right)=\lim _{n \rightarrow \infty} \operatorname{Tr}\left(P_{\Lambda} T_{n}^{k} P_{\Lambda}\right)
$$

However, $t_{\Delta_{n}}$ has been chosen to satisfy the identity

$$
\operatorname{Tr}\left(P_{\Lambda} T_{n}^{k} P_{\Lambda}\right)=\int_{\Delta_{n}^{k}} 1_{\Lambda}\left(x_{1}\right) t_{\Delta_{n}}\left(x_{1}, x_{2}\right) \cdots t_{\Delta_{n}}\left(x_{k}, x_{1}\right) \lambda\left(d x_{1}\right) \cdots \lambda\left(d x_{k}\right)
$$

and we have seen that $t_{\Delta_{n}}$ can be replaced by $t$ in this integral. Hence, statement (c) will be proved once we have shown that the right-hand side 
of (A.8) converges to the integrals in (c). For $k=1$, this is evident from the above. In the case $k=2$, (A.8) means that

$$
\operatorname{Tr}\left(P_{\Lambda} T_{n}^{2} P_{\Lambda}\right)=\int_{E^{2}} 1_{\Lambda}\left(x_{1}\right) 1_{\Delta_{n}}\left(x_{2}\right)\left|t\left(x_{1}, x_{2}\right)\right|^{2} \lambda\left(d x_{1}\right) \lambda\left(d x_{2}\right) .
$$

The integrand is non-negative and increases to $1_{\Lambda}\left(x_{1}\right)\left|t\left(x_{1}, x_{2}\right)\right|^{2}$ as $n \rightarrow \infty$. On the other hand, $\operatorname{Tr}\left(P_{\Lambda} T_{n}^{2} P_{\Lambda}\right) \leqslant\|T\| \operatorname{Tr}\left(P_{\Lambda} T_{n} P_{\Lambda}\right)=\|T\| \operatorname{Tr}\left(T_{\Lambda}\right)<$ $\infty$ for all $n$ with $\Delta_{n} \supset \Lambda$. The monotone convergence theorem thus shows that $1_{\Lambda}\left(x_{1}\right)\left|t\left(x_{1}, x_{2}\right)\right|^{2}$ is integrable on $E^{2}$. This proves statement (c) for $k=2$, and also assertion (b) by Fubini's theorem. Finally, let $k \geqslant 3$. Equation (A.8) can then be rewritten in the form

$$
\operatorname{Tr}\left(P_{\Lambda} T_{n}^{k} P_{\Lambda}\right)=\int_{\Lambda}\left(t_{x}, T_{n}^{k-2} t_{x}\right) \lambda(d x)
$$

and the integrand is bounded by $\left(t_{x}, T_{n}^{k-2} t_{x}\right) \leqslant\|T\|^{k-2}\left(t_{x}, t_{x}\right)$ uniformly in $n$. We also know from the case $k=2$ that this upper bound is locally integrable. We know further that $\left(t_{x}, T_{n}^{k-2} t_{x}\right)$ converges to $\left(t_{x}, T^{k-2} t_{x}\right)$ for all $x$ with $t_{x} \in L^{2}(E, \lambda)$, and thus for $\lambda$-a.a. $x$. Again, these functions are bounded by $\|T\|^{k-2}\left(t_{x}, t_{x}\right)$. Together with (A.7), this gives the trace formula in (c) for $k \geqslant 3$. The proof is therefore complete.

A final useful tool is the following projection-inversion lemma appearing without proof in (ref. 18, p. 18); see (ref. 25, Corollary 5.3) for the matrix version.

Lemma A.5. Let $T$ be any bounded positive operator with bounded inverse $T^{-1}$. Then for any projection $P$,

$$
P T^{-1} P \geqslant P(P T P)^{-1} P .
$$

Proof. Since $T$ is positive and invertible, any restriction of $T$ to some subspace is invertible in this subspace. This means that the operators $R:=P(P T P)^{-1} P$ and $S:=P^{\perp}\left(P^{\perp} T^{-1} P^{\perp}\right)^{-1} P^{\perp}$ are well defined, where $P^{\perp}=I-P$. The key observation is the decomposition formula

$$
P T^{-1} P=R+P T^{-1} S T^{-1} P .
$$

To see this we observe that $P^{\perp} T^{-1}\left(P^{\perp}+P\right) T P=0$. Multiplying with $S$ from the left we find $P^{\perp} T P=-S T^{-1} P T P$. Inserting this into the identity $P T^{-1}\left(P T P+P^{\perp} T P\right)=P$ we get

$$
P T^{-1}\left(I-S T^{-1}\right) P T P=P .
$$


Multiplying with $R$ from the right and rearranging we arrive at (A.9). As the second operator on the right-hand side of (A.9) is positive, the lemma follows.

\section{ACKNOWLEDGMENTS}

We are grateful to the referees whose remarks helped to improve this paper. H.J.Y. would like to thank Prof. T. Shirai and Prof. Y. Takahashi for inviting him to Kanazawa University. Part of this work was done during this stay. We also thank Prof. Y. M. Park for informing us about ref. 18. H.J.Y. was supported by Korea Research Foundation Grant (KRF-2002-015-CP0038).

\section{REFERENCES}

1. R. Bhatia, Matrix Analysis (Springer, New York, 1997).

2. A. Borodin, A. Okounkov, and G. Olshanski, Asymptotics of Plancherel measures for symmetric groups, J. Am. Math. Soc. 13(3), 481-515 (2000).

3. A. Borodin and G. Olshanski, Point processes and the infinite symmetric group, Part VI: Summary of results, available online at URL http://arxiv.org/abs/math.RT/9810015.

4. D. J. Daley and D. Vere-Jones, An Introduction to the Theory of Point Processes (Springer-Verlag, New York, 1988).

5. H.-O. Georgii and T. Küneth, Stochastic comparison of point random fields, J. Appl. Prob. 34:868-881 (1997).

6. E. Glötzl, Konstruktion der bedingten Energie eines Punktprozesses, Serdica 7:217-233 (1981).

7. G. Grimmett, Percolation, 2nd ed. (Springer, Berlin, 1999).

8. P. R. Halmos and V. S. Sunder, Bounded Integral Operators on $L^{2}$ Spaces (SpringerVerlag, Berlin, 1978).

9. Y. G. Kondratiev and T. Kuna, Correlation functions for Gibbs measures and Ruelle bounds, Methods Funct. Anal. Topology 9:9-58 (2003).

10. O. K. Kozlov, Gibbsian description of point random fields, Theory Prob. Appl. 21:339355 (1976).

11. R. Lyons, Determinantal probability measures, Publ. Math. Inst. Hautes Études Sci. 98:167-212 (2003).

12. R. Lyons and J. E. Steif, Stationary determinantal process: Phase multiplicity, Bernoullicity, entropy, and domination, Duke Math. J. 120(3), 515-575 (2003).

13. O. Macchi, The coincidence approach to stochastic point processes, Adv. Appl. Prob. 7:83-122 (1975).

14. K. Matthes, W. Warmuth, J. Mecke, Bemerkungen zu einer Arbeit von Nguyen Xuan Xanh und Hans Zessin, Math. Nachr. 88:117-127 (1979).

15. J. Mecke, Stationäre zufällige Maße auf lokalkompakten abelschen Gruppen, Z. Wahrscheinlichkeitstheorie verw. Geb. 9:36-58 (1967).

16. R. Meester and R. Roy, Continuum Percolation (Cambridge University Press, 1996).

17. X. X. Nguyen and H. Zessin, Integral and differential characterizations of the Gibbs process, Math. Nachr. 88:105-115 (1979).

18. M. Ohya and D. Petz, Quantum Entropy and its Use (Springer-Verlag, Berlin, 1993). 
19. F. Papangelou, The conditional intensity of general point processes and an application to line processes, Z. Wahrscheinlichkeitstheorie verw. Geb. 28:207-226 (1974).

20. C. J. Preston, Spatial birth-and-death processes, Bull. Inst. Int. Statist. 46:371-391 (1976).

21. C. J. Preston, Random Fields, Lecture Notes in Mathematics Vol. 534 (Springer Verlag, Berlin, 1976).

22. M. Reed and B. Simon, Methods of Modern Mathematical Physics II: Fourier Analysis, Self-Adjointness (Academic Press, New York, 1975).

23. H. Shimomura, Poisson measures on the configuration space and unitary representations of the group of diffeomorphisms, J. Math. Kyoto Univ. 34(3), 599-614 (1994).

24. T. Shirai and Y. Takahashi, Random point field associated with certain Fredholm determinant I: fermion, poisson, and boson point processes, J. Funct. Anal. 205:414-463 (2003).

25. T. Shirai and Y. Takahashi, Random point field associated with certain Fredholm determinant II: fermion shift and its ergodic and Gibbs properties, Ann. Prob. 31:1533-1564 (2003).

26. T. Shirai and H. J. Yoo, Glauber dynamics for fermion point processes, Nagoya Math. J. 168:139-166 (2002).

27. B. Simon, Trace Ideals and Their Applications (Cambridge University Press, Cambridge, 1979).

28. A. Soshnikov, Determinantal random point fields, Russ. Math. Surv. 55:923-975 (2000).

29. H. Spohn, Interacting brownian particles: A study of Dyson's model, in, Hydrodynamic behaviour and interacting particle systems (Minneapolis, Minn., 1986), G. Papanicolaou, ed., IMA Vol. Math. Appl. 9 (1987).

30. H. Spohn, Tracer dynamics in Dyson's model of interacting brownian particles, J. Stat. Phys. 47:669-679 (1987).

31. V. Strassen, The existence of probability measures with given marginals, Ann. Math. Statist. 36:423-439 (1965).

32. H. J. Yoo, Gibbsianness of fermion random point fields, Preprint (2003). 